\section{بررسى تأثير مداخلات ارتونومى در فعاليتهاى دستى بر كاهش اختلالات اسكلتى وعضلانى به روش}

\author{
سارا دهنوى '، عبداله واحدى ؟َ"، مجيد معتمدزاده طرقبه "، عباس مقيم \\ بيگى
}

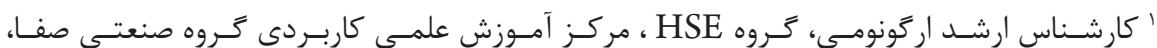

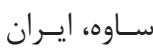

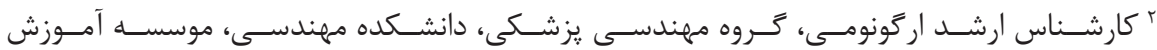

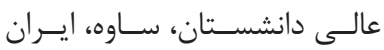

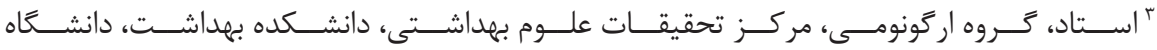

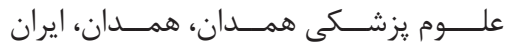

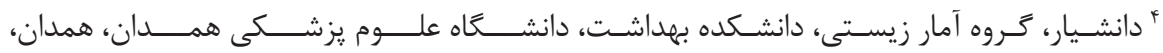
ائران

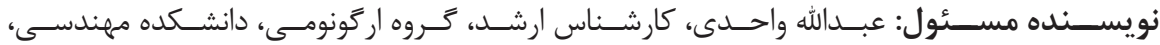

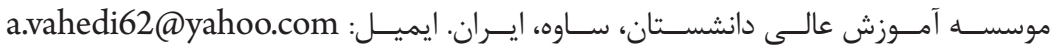

جمكيده

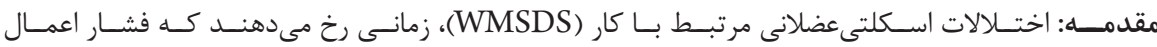

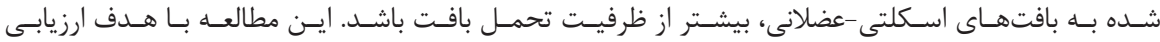

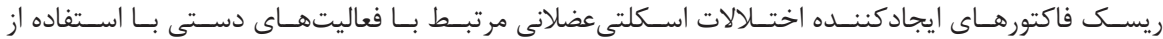

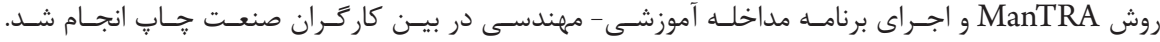

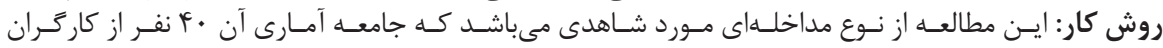

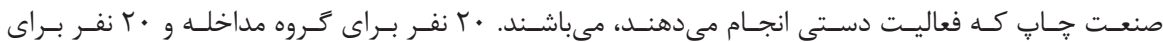

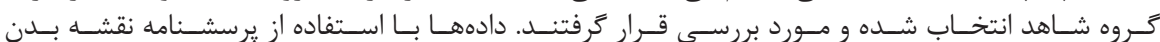

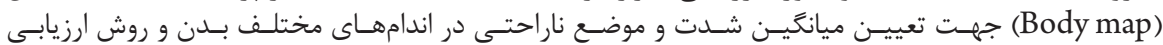
جهanTRA

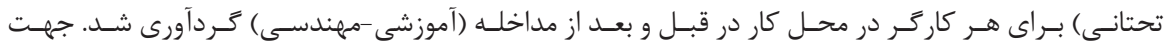

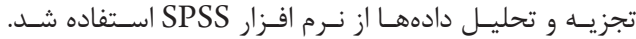

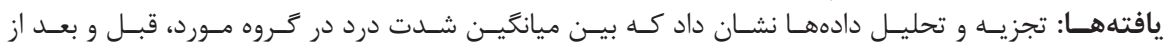

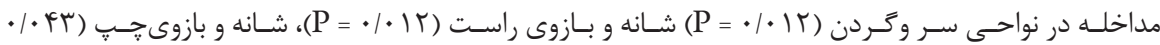

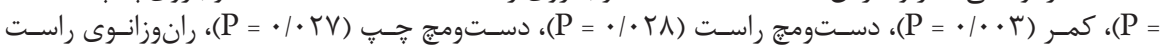

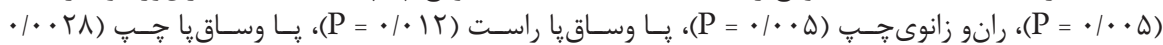

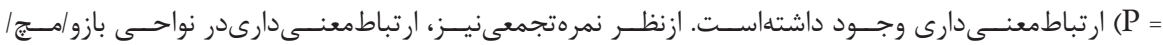

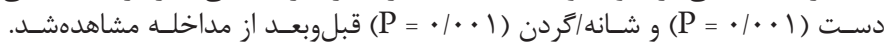

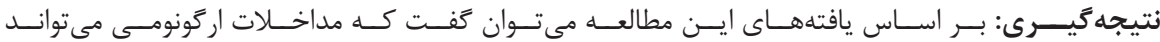

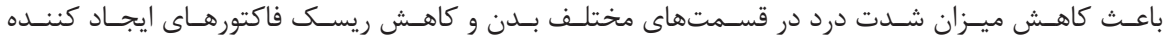

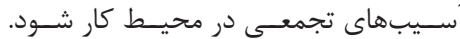

DOI: $10.21859 /$ joe- 04048

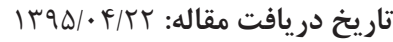

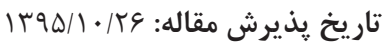

وازَّان كليدى: اختلالات اسكلتى عضلانى كليدى:

ManTRA مداخلات ارگونوميكى وظايف دستى مداخلات Body map

تممامى حقوق نشر براى دانشكاه علوم يزشكى

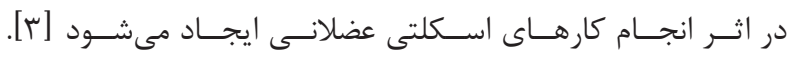

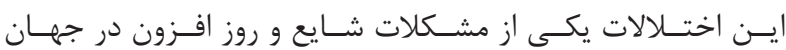

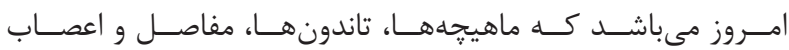

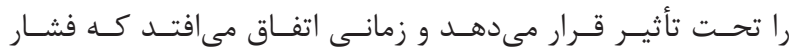

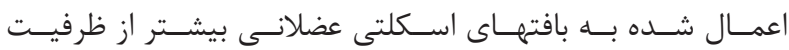

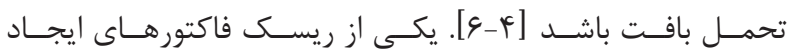

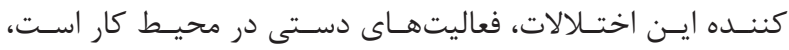

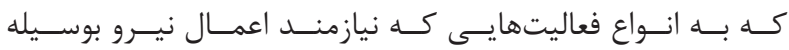

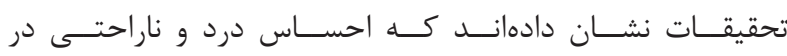

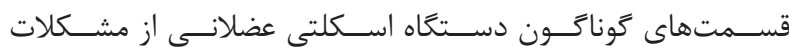

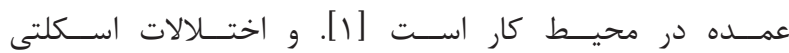

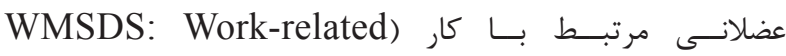
فusculoskeletal Disorders

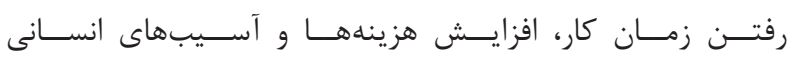

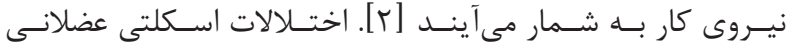

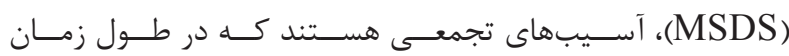




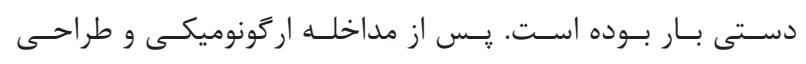

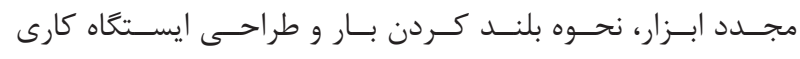

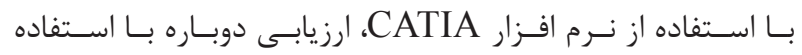

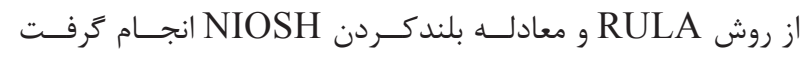

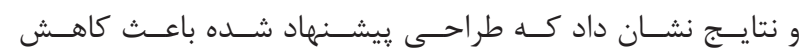

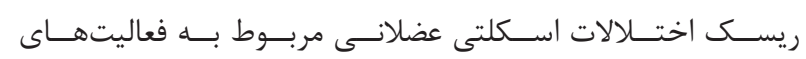

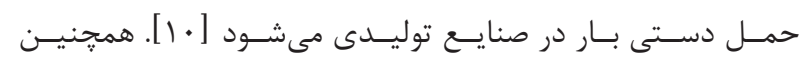

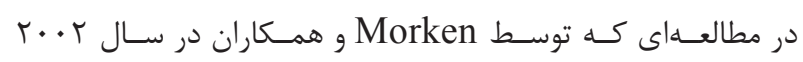

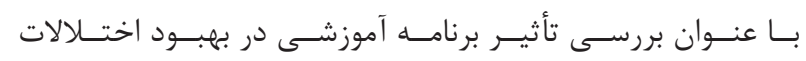

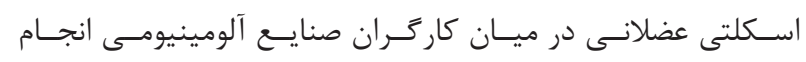

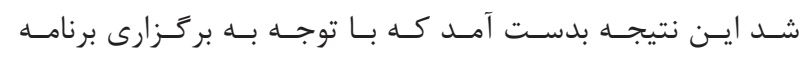

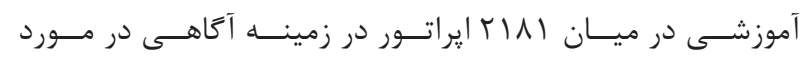

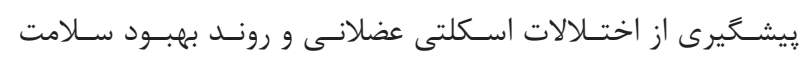

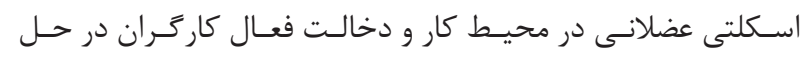

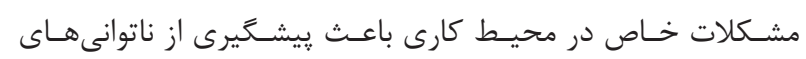

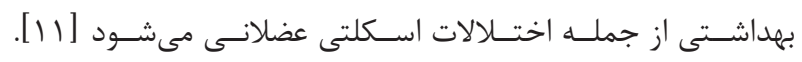

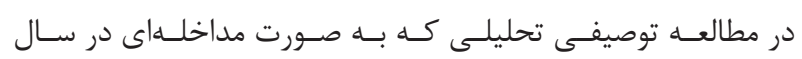

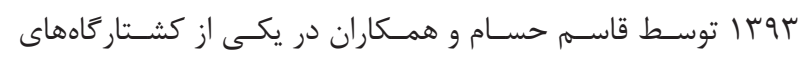

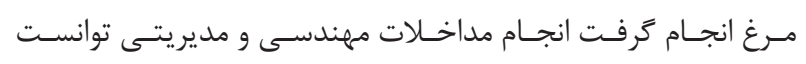

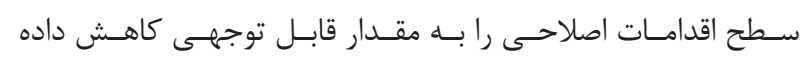

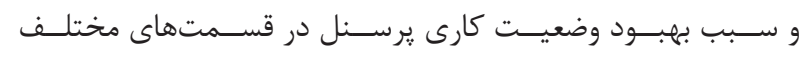

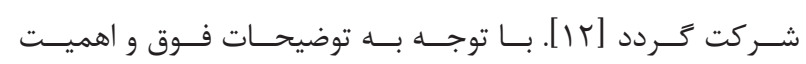

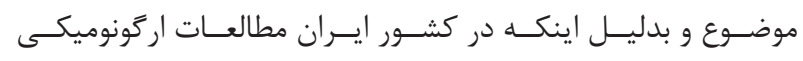

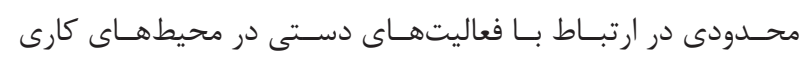

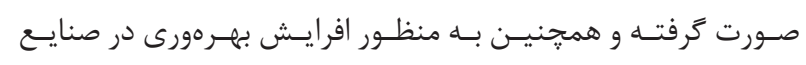

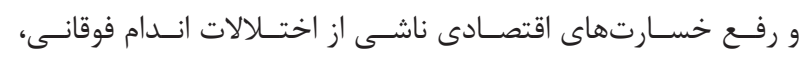

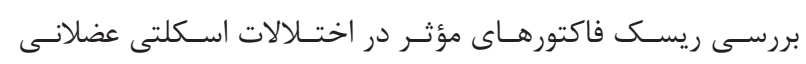

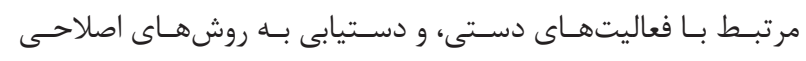

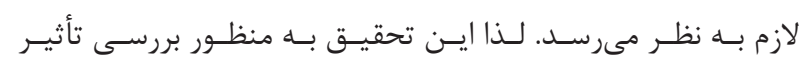

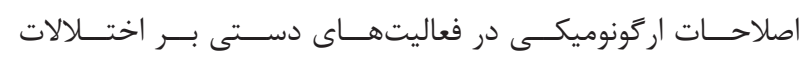

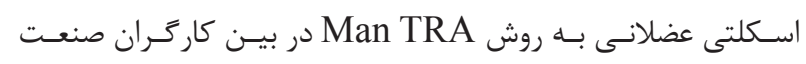

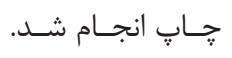

روش كار

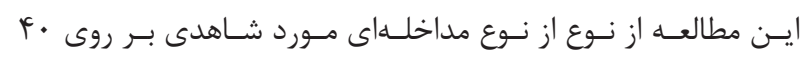

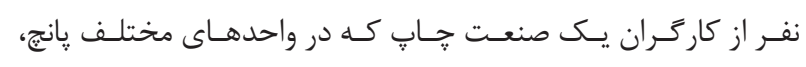

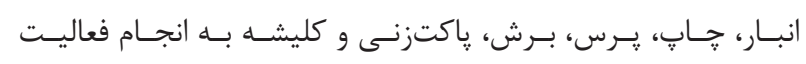

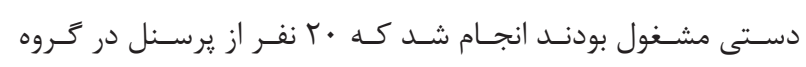

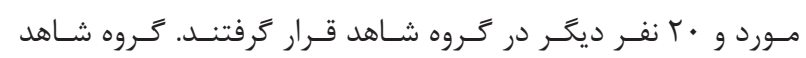

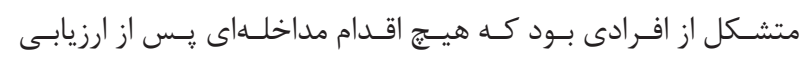

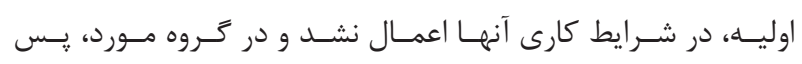

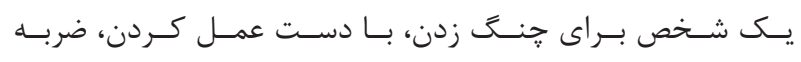

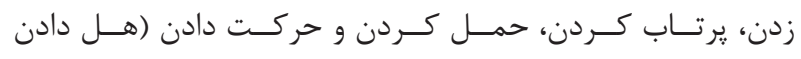

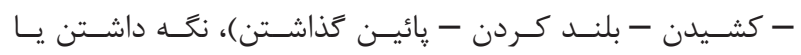

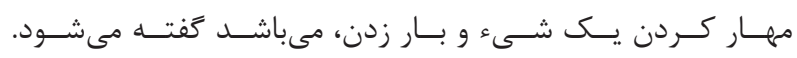

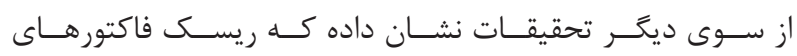

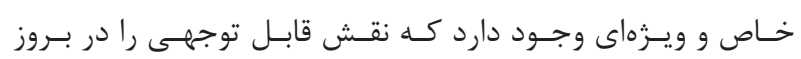

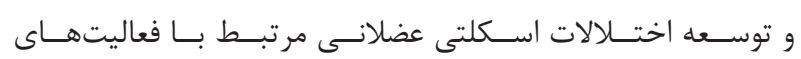

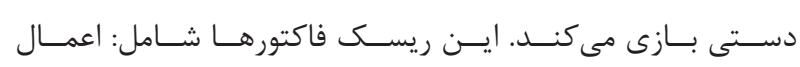

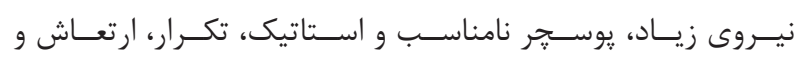

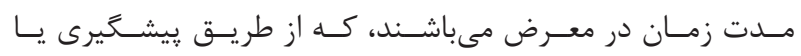

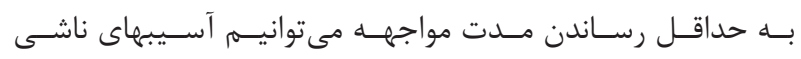

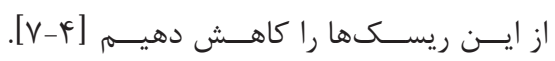

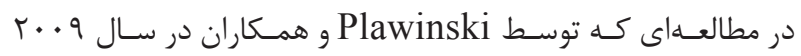

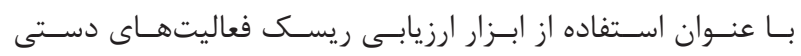

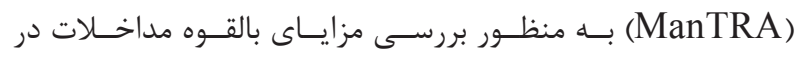

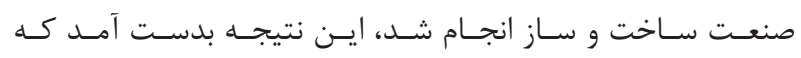

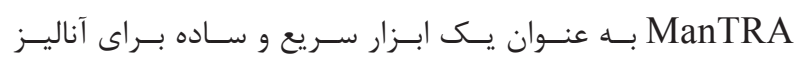

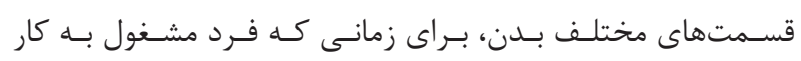

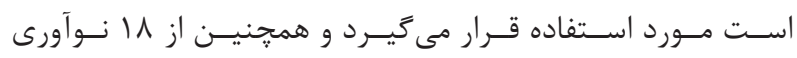

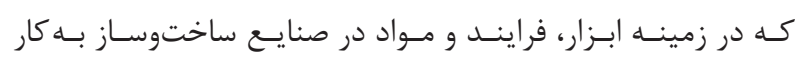

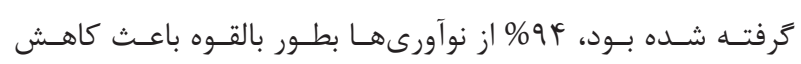

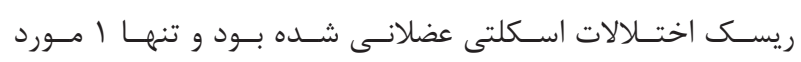

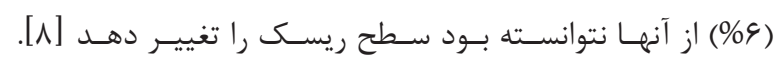

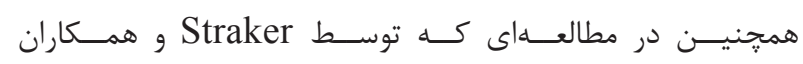

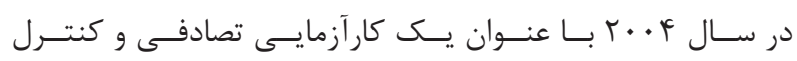

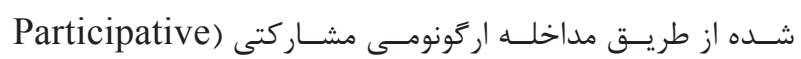
من ergonomics intervention

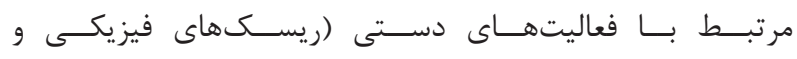

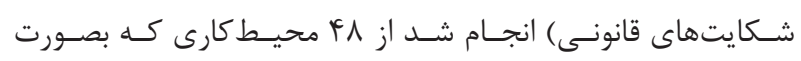

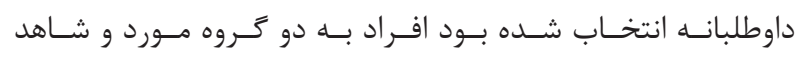

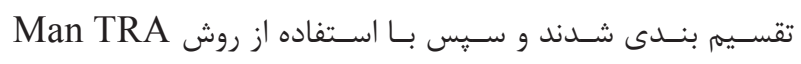

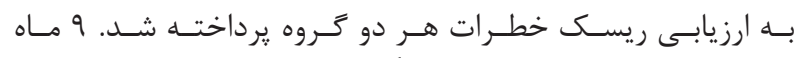

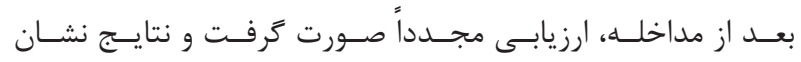

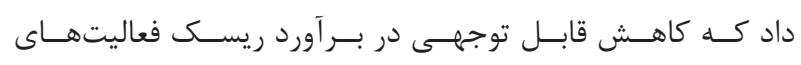

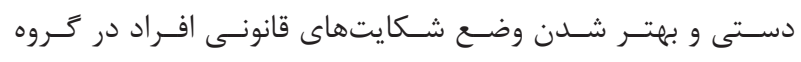

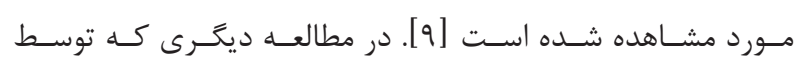
ال دia Huey sky

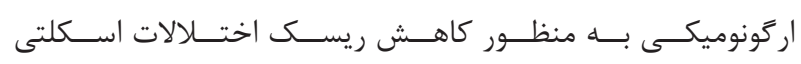

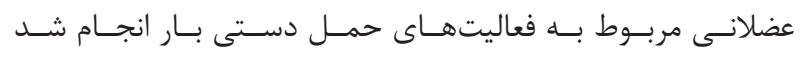

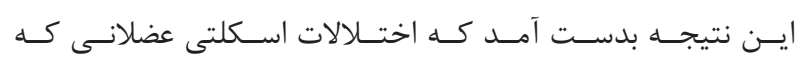

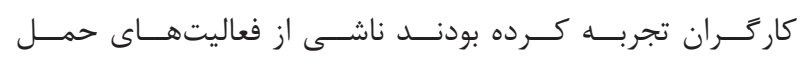




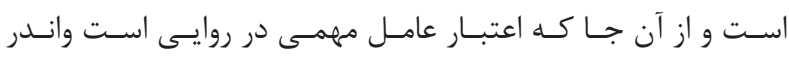

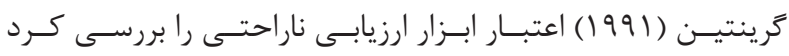

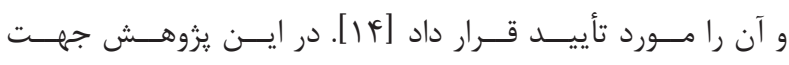

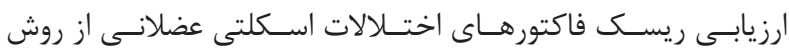

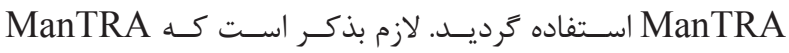

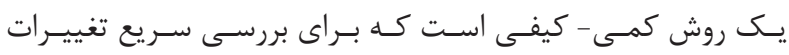

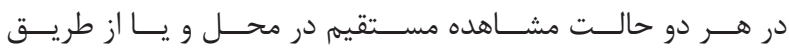

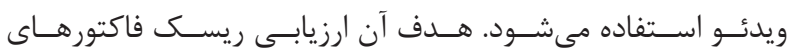

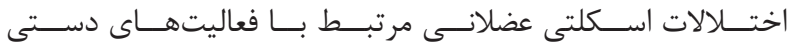

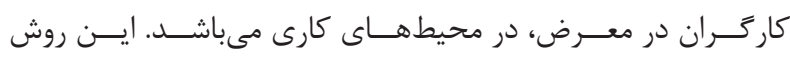

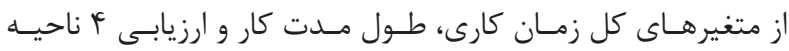

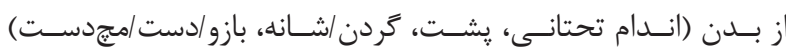

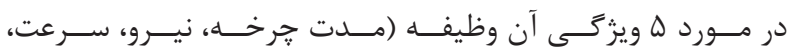

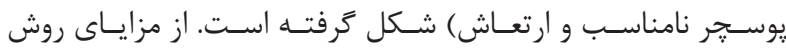

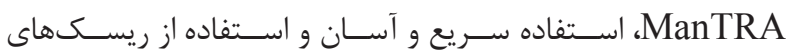
عمومسى و همخانسى فعاليتهـاى جابجايسى مـواد (بــهـ عنــوان مثــال:

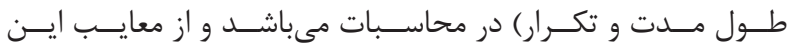

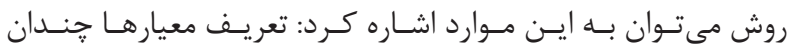

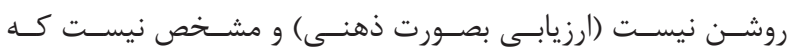

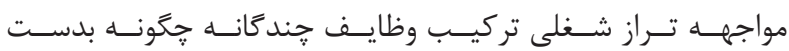
مى آيـد [10.

\section{فاز دوم: اجراى برنامه مداخله آموزشى - مهيندسى}

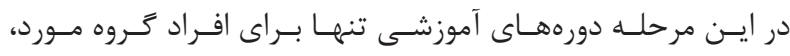

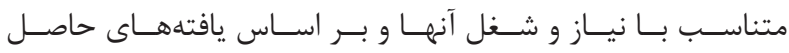

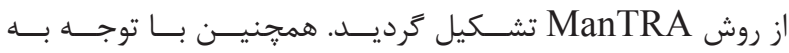

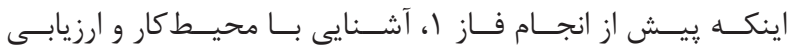

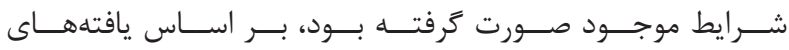

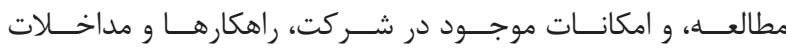

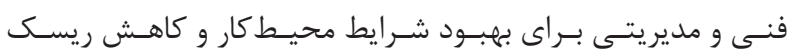

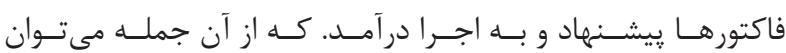

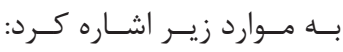

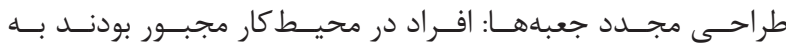

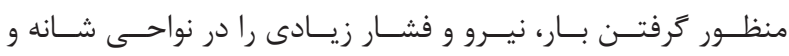

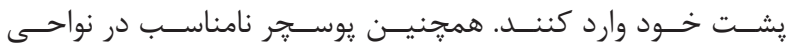

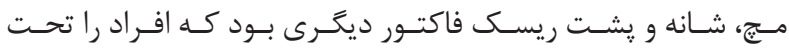

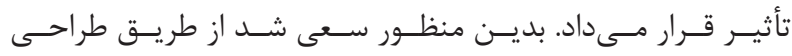

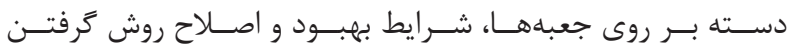

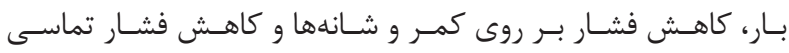

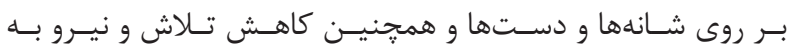
منظـــور انجـام وظايـف كارى مهيــا شـود.

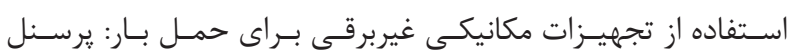

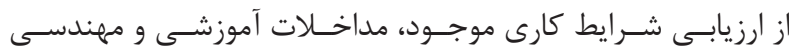

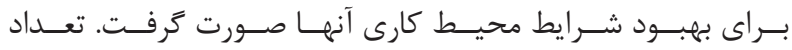

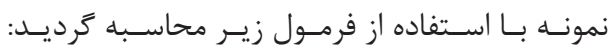
$\mathrm{n}=\left(\left(2 \sigma^{2}\right)\left(\mathrm{z}_{1-\alpha / 2}+\mathrm{z}_{1-\beta}\right)^{2}\right) / \mathrm{d}^{2}$

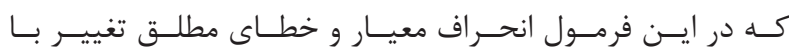

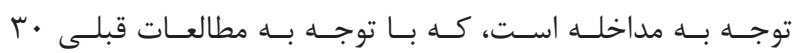

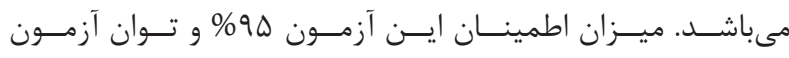

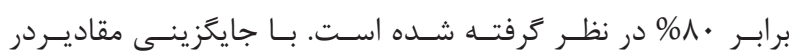

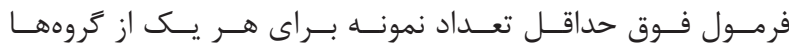

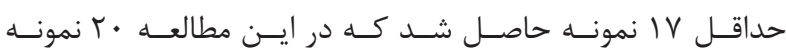

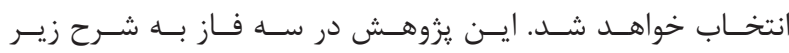

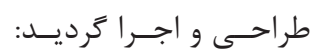

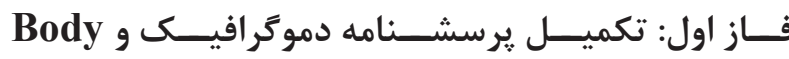

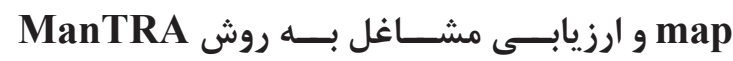

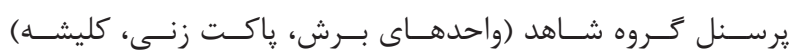

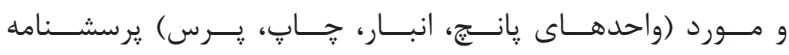

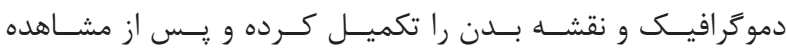

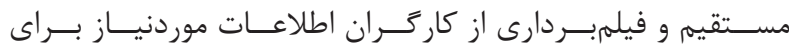

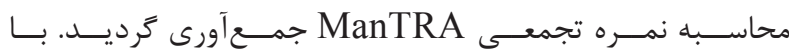

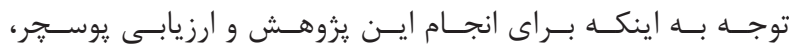

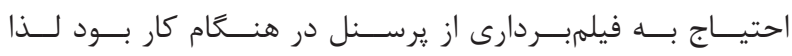

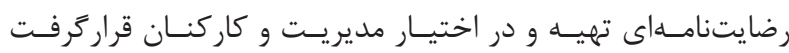

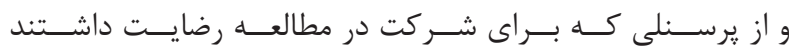

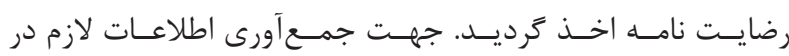

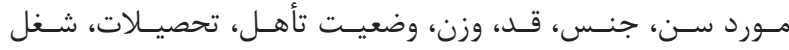

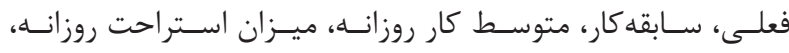

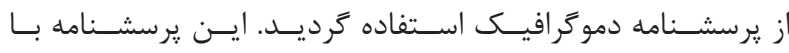

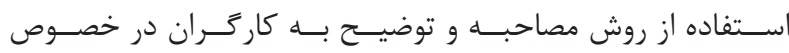

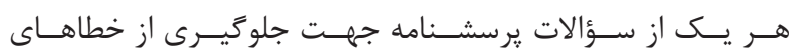

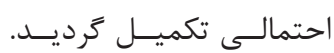

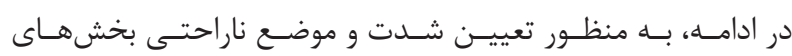

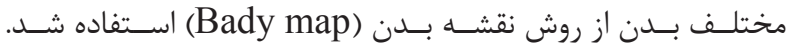

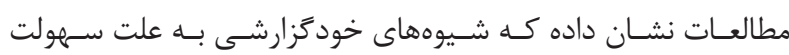

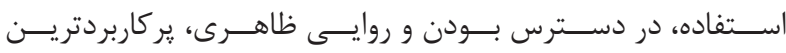

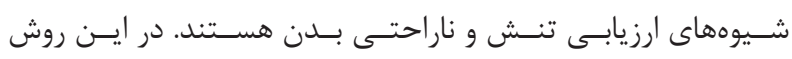

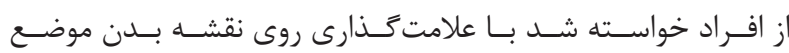

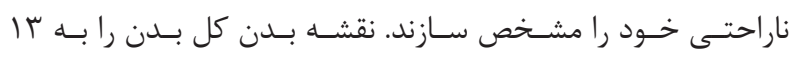

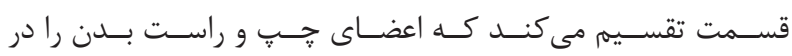

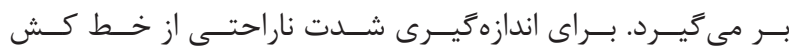

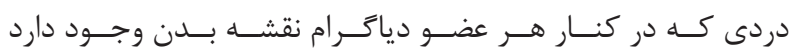

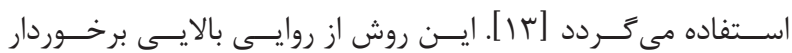




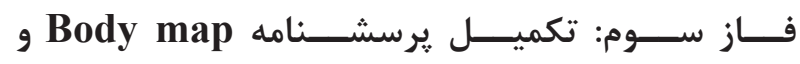

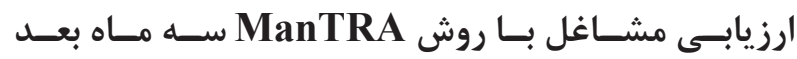

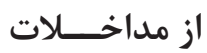

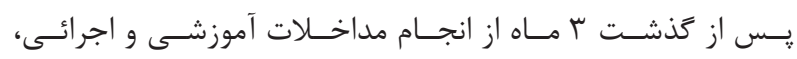

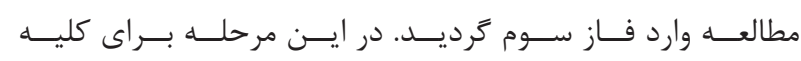

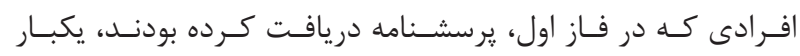

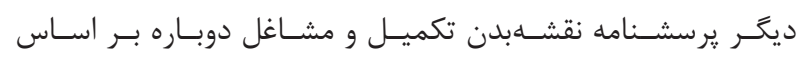

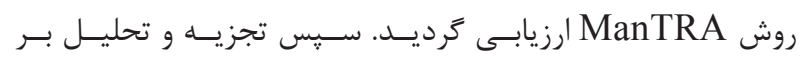

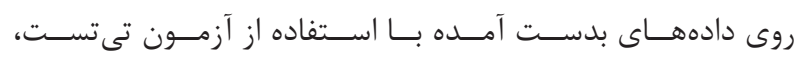

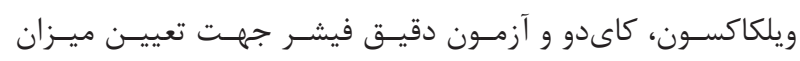

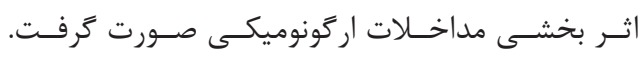

\section{روش تجزيه و تحليل دادهها}

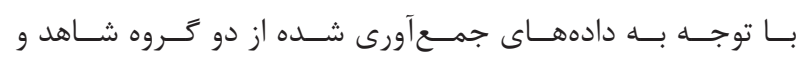

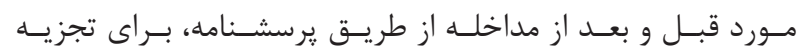

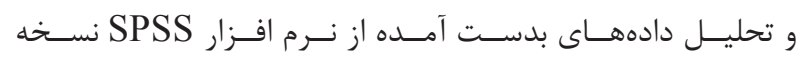

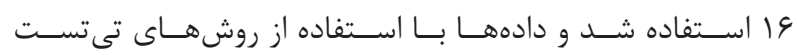

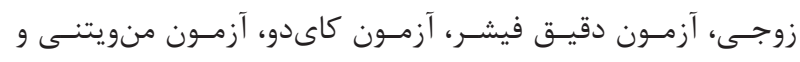

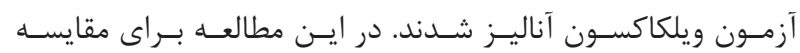

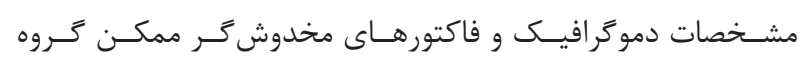

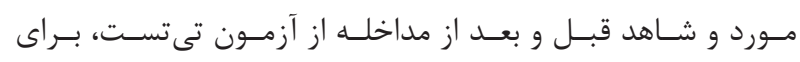

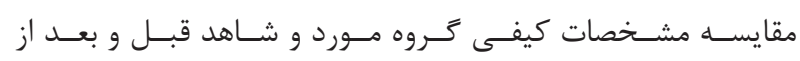

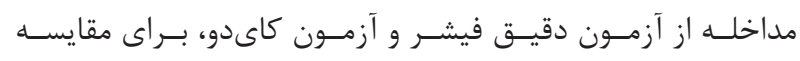

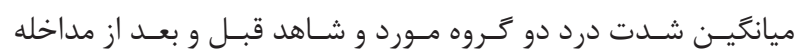

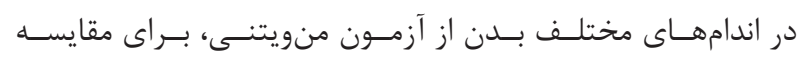

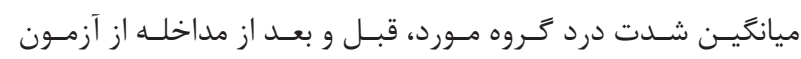

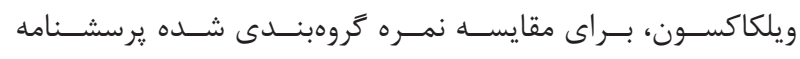

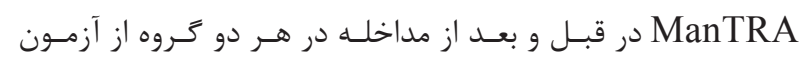

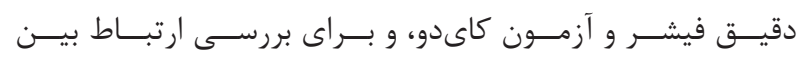

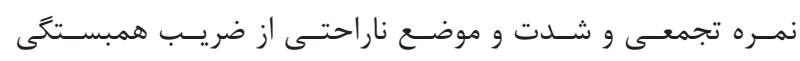

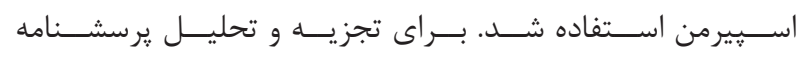
ManTRA

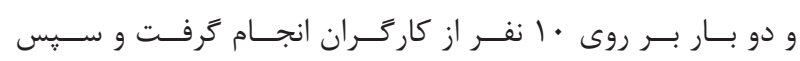

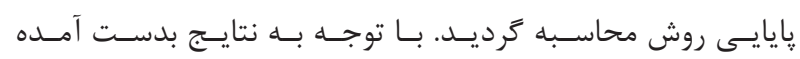

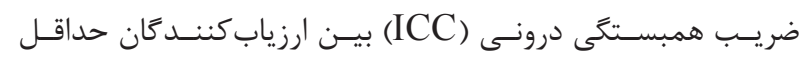

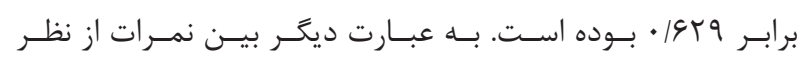

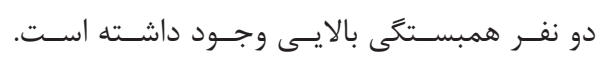

\section{يافتهها}

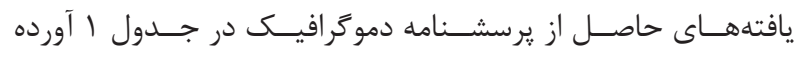

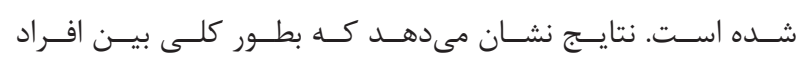

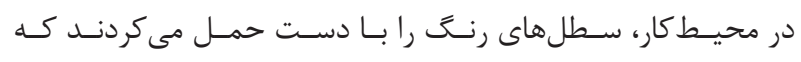

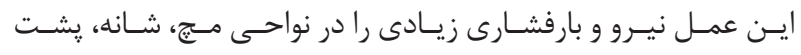

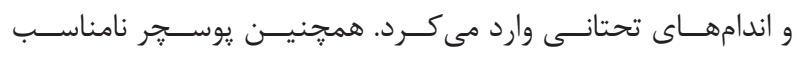

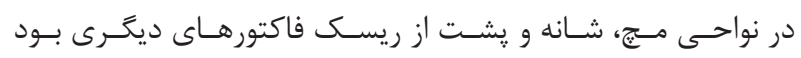

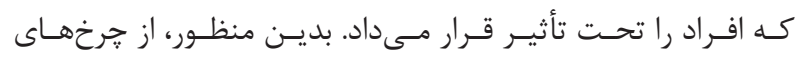

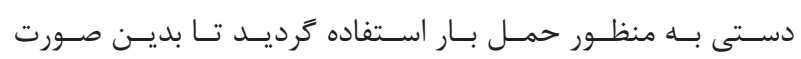

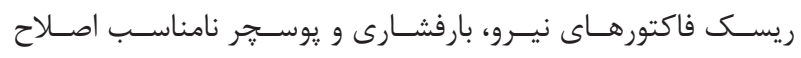
كَردد.

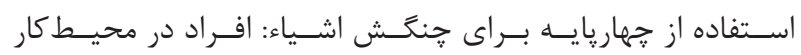

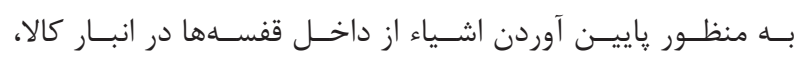

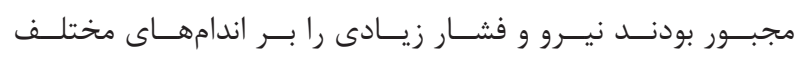

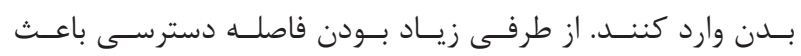

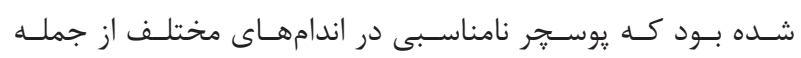

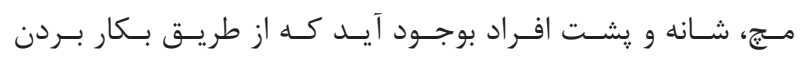

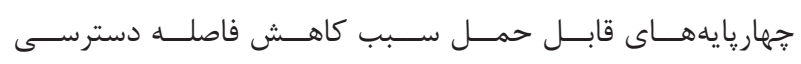

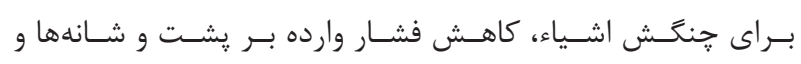

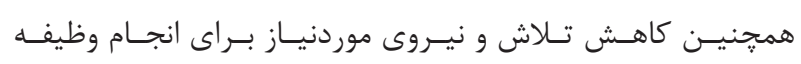

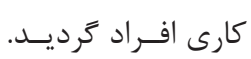

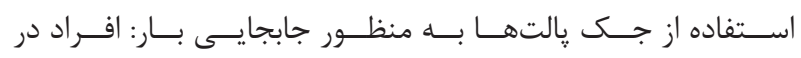

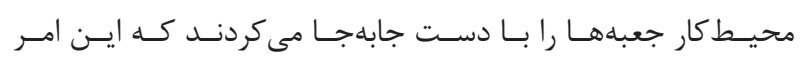

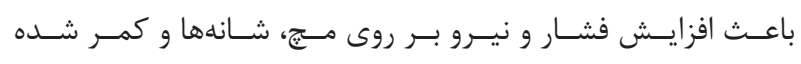

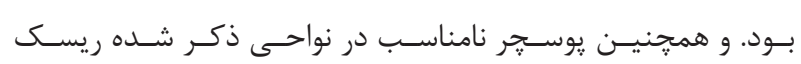

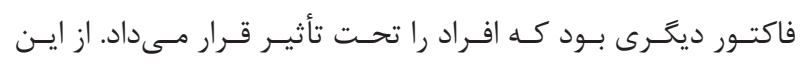

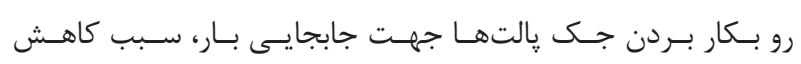

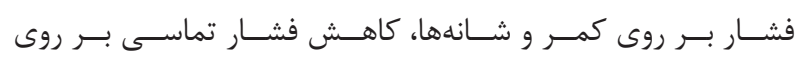

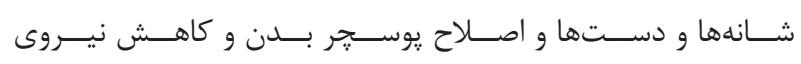

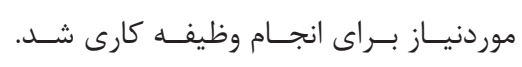

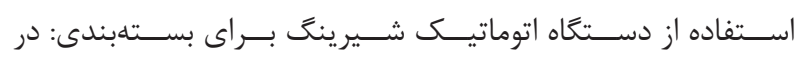

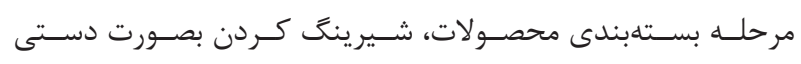

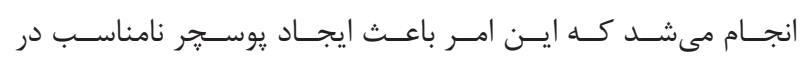

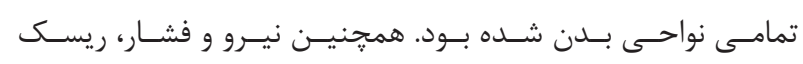

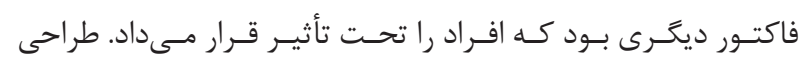

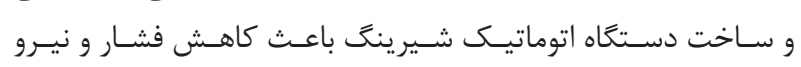

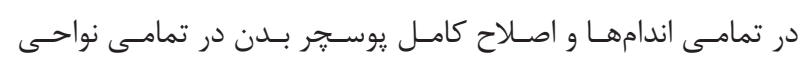

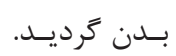

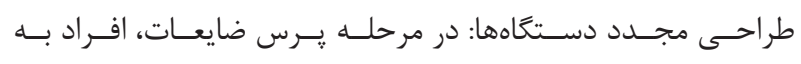

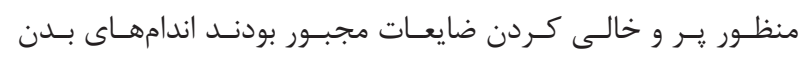

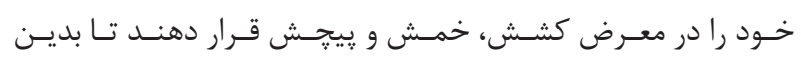

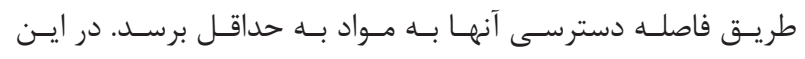

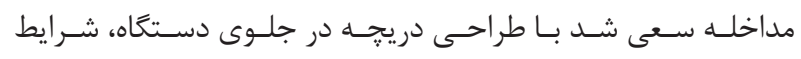

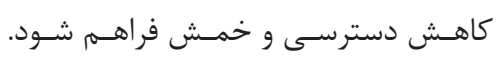




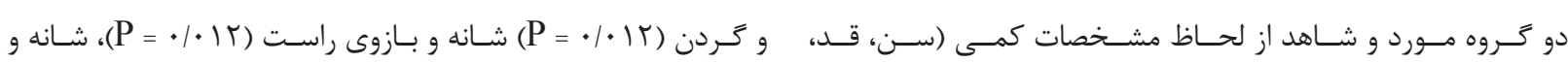

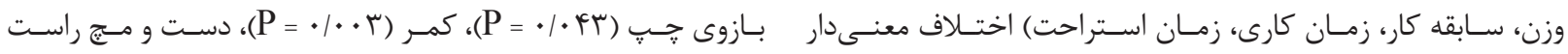

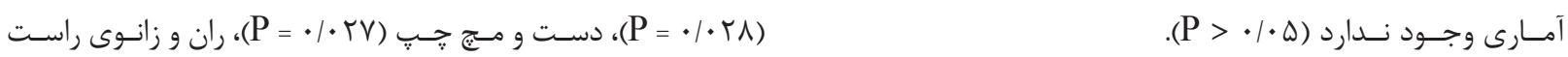

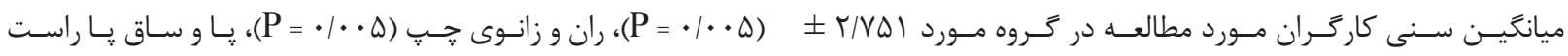

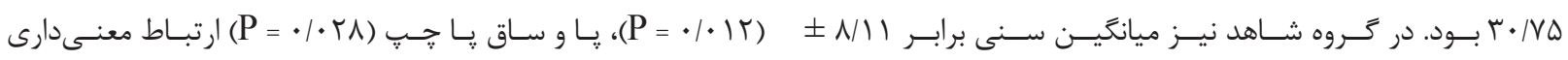

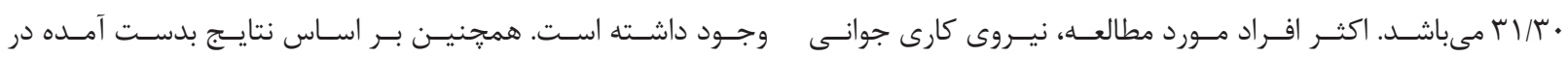

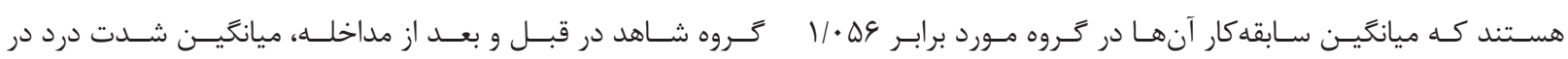

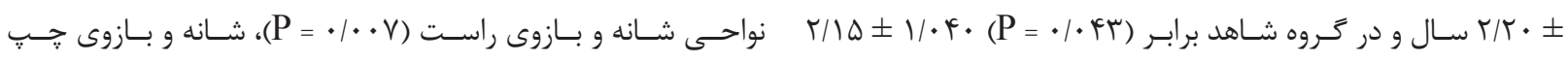

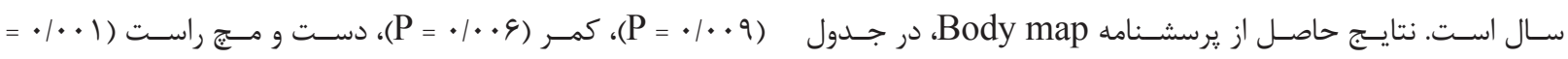

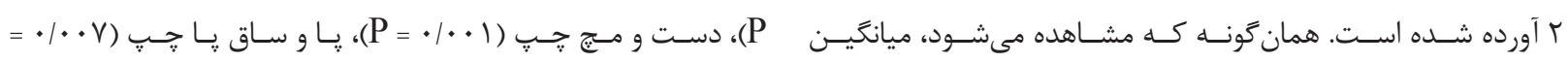

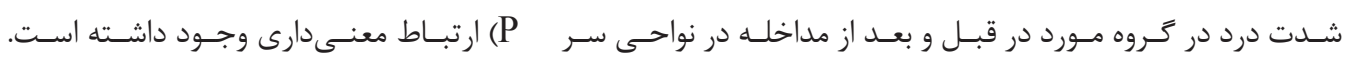

\begin{tabular}{|c|c|c|c|c|}
\hline \multirow[b]{2}{*}{ Df } & \multirow[b]{2}{*}{ t } & \multicolumn{3}{|c|}{ جدول ا: مقايسه مشخصات دموكرافيك كروه مورد و شاهد } \\
\hline & & انحراف معيار 土 ميانتين & 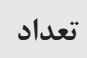 & مورد - شاهد \\
\hline \multirow[t]{3}{*}{$\mathrm{Df}=r \wedge$} & $\mathrm{P}=\cdot / V \vee \varphi, \mathrm{t}=-\cdot / r \wedge \vee$ & & & سن \\
\hline & & $r \cdot / V \Delta \pm r / V \Delta$ & $r \cdot$ & مورد \\
\hline & & $\Gamma / \Gamma \cdot \pm N / 11$ & r. & 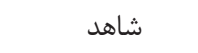 \\
\hline \multirow[t]{3}{*}{$\mathrm{Df}=r v$} & $\mathrm{P}=\cdot|\Delta \mu G, \mathrm{t}=\cdot| G T F$ & & & قد \\
\hline & & $|\vee G| G \Lambda \pm \Delta / r \Lambda \varepsilon$ & 19 & مورد \\
\hline & & $\mid V \Delta / F \Delta \pm \varepsilon / A T r$ & $r \cdot$ & شاهد \\
\hline \multirow[t]{3}{*}{$\mathrm{Df}=\mu_{\varphi}$} & $\mathrm{P}=\cdot / \& \varepsilon \cdot, \mathrm{t}=-\cdot / V \psi V$ & & & 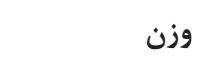 \\
\hline & & $V G||| \pm 1 \cdot| g r \mid$ & 11 & مورد \\
\hline & & $V N / G \Lambda \pm 1 \cdot \mid \Delta T K$ & $r \cdot$ & 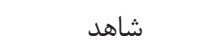 \\
\hline \multirow[t]{3}{*}{$\mathrm{Df}=r \wedge$} & $\mathrm{P}=\cdot|\Lambda \Lambda|, \mathrm{t}=\cdot|| \Delta \mid$ & & & سابقه كار \\
\hline & & $r / T \cdot \pm 1 / \cdot \Delta \varphi$ & $r \cdot$ & مورد \\
\hline & & $r /\|\Delta \pm\| \cdot r$ & $r$. & 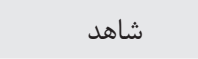 \\
\hline \multirow[t]{3}{*}{$\mathrm{Df}=r \wedge$} & $\mathrm{P}=\cdot / \mu T F, \mathrm{t}=-1 / \cdots$ & & & زمان كارى \\
\hline & & $\| / F \Delta \pm r / F \varphi$. & r. & مورد \\
\hline & & $\mid r / \cdots \pm \cdot / \cdots$ & $r$. & شاهد \\
\hline \multirow{3}{*}{\multicolumn{2}{|c|}{ زمان استراحت در دو كروه كاملاً يكسان بوده است كه آزمون t براى آن انجام }} & & & 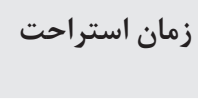 \\
\hline & & $1 / \cdots \pm \cdot / \cdots$ & $r \cdot$ & 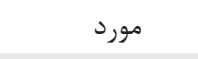 \\
\hline & & $1 / \cdots \pm \cdot / \cdots$ & $r$. & شاهد \\
\hline
\end{tabular}




\begin{tabular}{|c|c|c|c|}
\hline \multirow[b]{2}{*}{ آزمون ويلكاكسون } & \multicolumn{3}{|c|}{ جدول r: مقايسه ميانگَين شدت درد، دو كروه مداخله و شاهد قبل و بعد از مداخله } \\
\hline & بعد از مداخله & قبل از مداخله & نواحى بدن \\
\hline & & & سر و عردن \\
\hline$Z=-r|\Delta r|, P=\cdot / \cdot \mid r$ & $I V / \Delta r$ & 19/4 & مورد \\
\hline \multirow[t]{2}{*}{$\mathrm{Z}=-\cdot / \cdot \Delta 9 ، \mathrm{P}=\cdot / 9 \Delta \mu$} & $r r / 4 \wedge$ & $r \mid / 9 \Lambda$ & 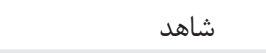 \\
\hline & & & 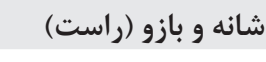 \\
\hline$Z=-r|\Delta r| ، P=\cdot / \cdot \mid r$ & $\mid k / T$. & $I V / \Delta \cdot$ & 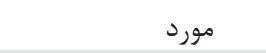 \\
\hline \multirow{2}{*}{$Z=-r / 999 ، \mathrm{P}=\cdot / \cdots V$} & r\&/A. & $r r / Q$. & 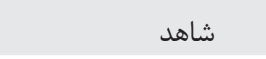 \\
\hline & & & شانه و بازو (جي) \\
\hline$Z=-r / \cdot r r \cdot P=\cdot / \cdot r r$ & $\mid \psi / \cdot r$ & $I V / T \cdot$ & 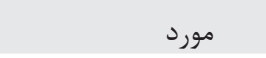 \\
\hline \multirow[t]{2}{*}{$Z=-r|q| r \cdot P=\cdot / \cdot 9$} & $r 9 / 9 \Lambda$ & $r$ r/V. & شاهد \\
\hline & & & كمر \\
\hline$Z=-r / q \mu r{ }_{d} P=\cdot / \cdot r$ & $\mid Q / q r$ & $19 / \mathrm{Vr}$ & مورد \\
\hline \multirow[t]{2}{*}{$Z=-r / V M 1 ، P=\cdot 1 \cdot \varphi \varphi$} & $r \Delta / r \Lambda$ & $r I / T \Lambda$ & شاهد \\
\hline & & & آرنج و ساعد (راست) \\
\hline$Z=-1 / 9 \cdot r \cdot P=\cdot / 1 \cdot 9$ & IV/A & $1 \Lambda / \Delta \Delta$ & مورد \\
\hline \multirow{2}{*}{$\mathrm{Z}=-\cdot / V \mu F \cdot \mathrm{P}=\cdot / 4 \& \mu$} & $r \mu / 1 \Lambda$ & $T r / F \Delta$ & 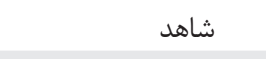 \\
\hline & & & آرنج و ساعد (جب) \\
\hline$Z=-1 / 9 \cdot 4 \cdot P=\cdot 11 \cdot 9$ & 19190 & $I V / G Y$ & مورد \\
\hline \multirow{2}{*}{$Z=-1 / 099 ، P=\cdot / 11$} & $r F / T \Delta$ & rr/ru & شاهد \\
\hline & & & دست و مج (راست) \\
\hline$Z=-r / r \cdot 1 ، P=\cdot / \cdot r \Lambda$ & $1 \% / 91$ & $19 / V \Delta$ & مورد \\
\hline \multirow{2}{*}{$\mathrm{Z}=-r / 4 \varepsilon \Delta, \mathrm{P}=\cdot / \cdot \cdot 1$} & $r N \cdot r$ & $T I / T \Delta$ & شاهد \\
\hline & & & دست و مج (ج)) \\
\hline$Z=-r / r \cdot V$. $P=\cdot / \cdot r V$ & $1 \% / 90$ & $11 / 4$ & مورد \\
\hline \multirow{2}{*}{$Z=-r / r \Delta r . P=\cdot / \cdot 1$} & TN/TM & Tr/A. & شاهد \\
\hline & & & باسن \\
\hline $\mathrm{Z}=-1 / \cdots \cdot \mathrm{P}=\cdot / \mu \mid \mathrm{V}$ & $r \cdot \mid \Delta r$ & $1 \wedge / \Delta \Lambda$ & مورد \\
\hline \multirow[t]{2}{*}{$Z=-1 / \wedge r \varsigma ، P=\cdot / \cdot \varphi \wedge$} & $r \cdot / 4 \Lambda$ & Tt/KT & شاهد \\
\hline & & & ران و زانو (راست) \\
\hline$Z=-r / \Lambda \cdot r \cdot P=\cdot / \cdot \Delta$ & $I V / F$ & $19 / 10$ & مورد \\
\hline \multirow[t]{2}{*}{$Z=-\cdot / \Delta r F \cdot P=\cdot \mid \Delta q r$} & $r$ r/g. & $r I / A \Delta$ & 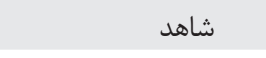 \\
\hline & & & ران و زانو (جֶ) \\
\hline$Z=-r / \Lambda \cdot r \cdot P=\cdot / \cdot \Delta$ & $10 / T r$ & IV/TK & مورد \\
\hline \multirow[t]{2}{*}{$\mathrm{Z}=-\cdot 199 \mathrm{~V}, \mathrm{P}=\cdot 10 \cdot \Delta$} & $r \Delta / V \Lambda$ & rr/vi & 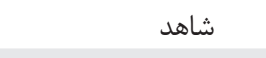 \\
\hline & & & يا و ساق ها (راست) \\
\hline $\mathrm{Z}=-r|\Delta r| ، \mathrm{P}=\cdot / \cdot \mid r$ & $1 V / 9 \Delta$ & $19 / \cdot 0$ & 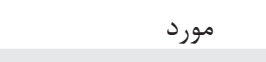 \\
\hline \multirow[t]{2}{*}{$Z=-|/ V Y| ، P=\cdot / \cdot \wedge \Delta$} & $r r / \cdot \Delta$ & $r I / 9 \Delta$ & 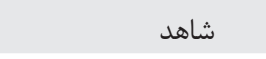 \\
\hline & & & 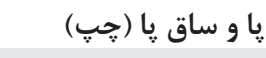 \\
\hline$Z=-r / r \cdot 1 ، P=\cdot / \cdot r \Lambda$ & $I V / T \Delta$ & $19 / 14$ & 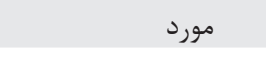 \\
\hline $\mathrm{Z}=-1 / 9 \wedge \Lambda ، \mathrm{P}=\cdot / \cdot r \Lambda$ & $r r / v \Delta$ & TI/AN & شاهد \\
\hline
\end{tabular}




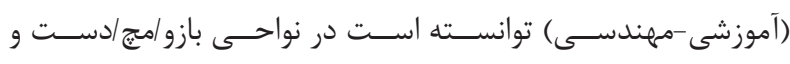

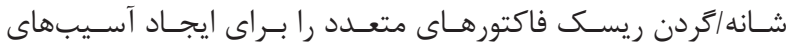

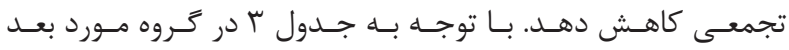

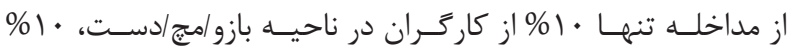

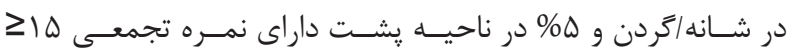

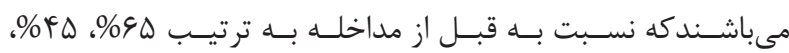

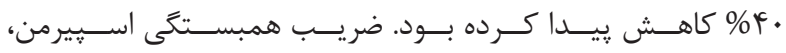

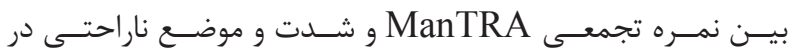

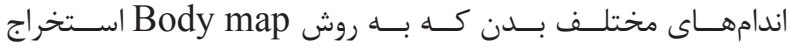

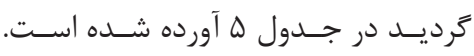

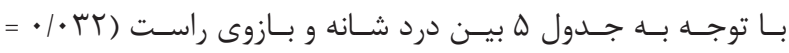

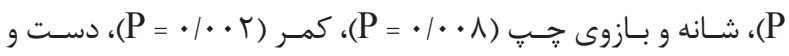

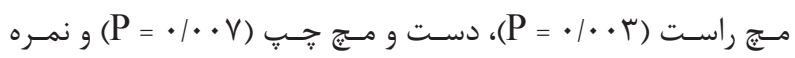
تجمعسى در ناحيـه مج/دست /بازو بعـد از مداخلـه ارتبـاط معنسى دار

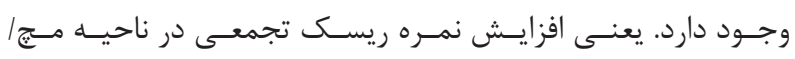

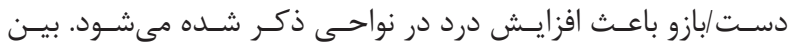

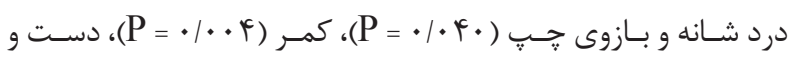

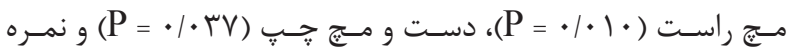

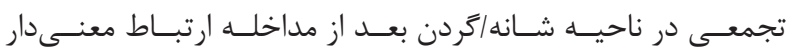

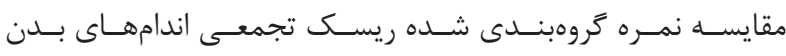

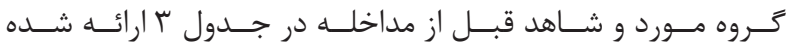

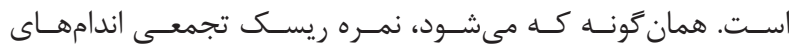

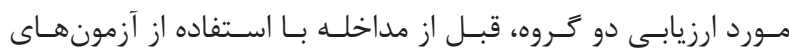

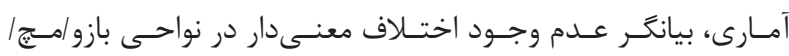

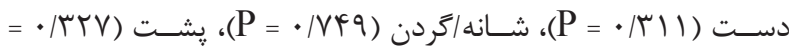

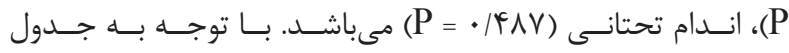

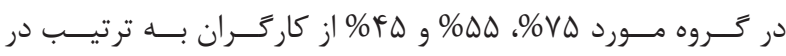

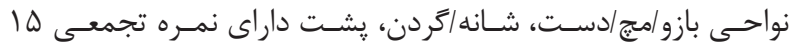

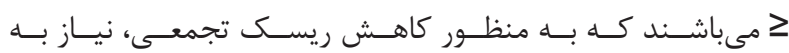

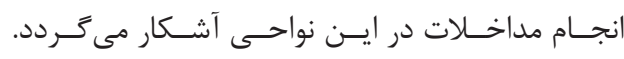

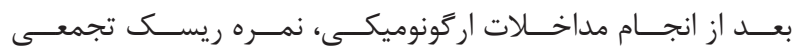

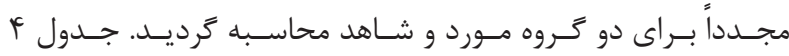

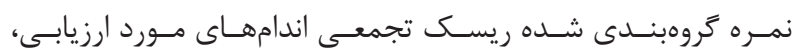

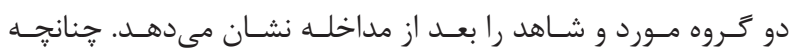

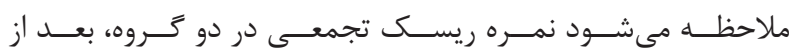

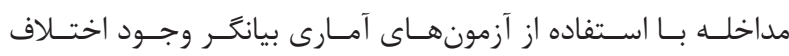

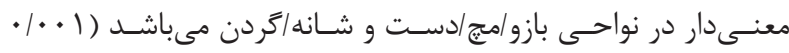
P =

\begin{tabular}{|c|c|c|c|c|c|}
\hline \multirow{4}{*}{ آزمون كاىدو - آزمون دقيق فيشر } & \multicolumn{5}{|c|}{ جدول با: مقايسه نمره كروهبندى شده ريسك تجمعى اندامهاى بدن گروه مورد و شاهد قبل از مداخله } \\
\hline & \multicolumn{4}{|c|}{ نمره كروهبندى ريسك تجمعى" } & نواحى بدن \\
\hline & \multicolumn{2}{|c|}{$1 \Delta \geq$} & \multicolumn{2}{|c|}{$10<$} & \\
\hline & شاهد & مورد & شاهد & مورد & \\
\hline$P=\cdot r / 11$ / & $\mid r(\varphi \cdot)$ & $1 \Delta(V \Delta)$ & $\Lambda(\psi \cdot)$ & $\Delta(Y \Delta)$ & بازو/مج/دست \\
\hline$P=\cdot / V \& q$, و مقدار آماره & $\mid r(\varphi \cdot)$ & $\|(\Delta \Delta)$ & $\Lambda\left(\varphi^{*} \cdot\right)$ & $q(\uparrow \Delta)$ & 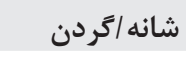 \\
\hline$P=\cdot / T r V$ و مقدار آماره & $\varphi(r \cdot)$ & $q(\varphi \Delta)$ & $\mid f(V \cdot)$ & $\|(\Delta \Delta)$ & يشت \\
\hline $\mathrm{P}=\cdot / \uparrow \wedge V$ & $r(1 \cdot)$ & $\cdot(\cdot)$ & $1 \wedge(9 \cdot)$ & $r \cdot(1 \cdots)$ & 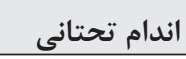 \\
\hline
\end{tabular}

" مقادير جدول به صورت تعداد (درصد) بيان شده است.

\begin{tabular}{|c|c|c|c|c|c|}
\hline \multicolumn{6}{|c|}{ جدول f: مقايسه نمره گروهبندى شده ريسك تجمعى اندامهاى بدن گروه مورد و شاهد بعد از مداخله } \\
\hline \multirow[t]{3}{*}{ آزمون كاىدو - آزمون دقيق فيشر } & \multicolumn{4}{|c|}{ نمره كروهبندى ريسك تجمعى " } & نواحى بدن \\
\hline & \multicolumn{2}{|c|}{$10 \geq$} & \multicolumn{2}{|c|}{$10<$} & \\
\hline & شاهد & مورد & شاهد & مورد & \\
\hline 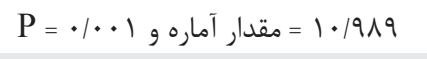 & $\mid r(\varphi \cdot)$ & $r(1 \cdot)$ & $(\boldsymbol{F} \cdot) \Lambda$ & $1 \wedge(9 \cdot)$ & 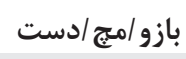 \\
\hline 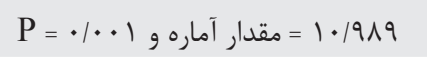 & $\operatorname{lr}(\varphi \cdot)$ & $r(1 \cdot)$ & $\Lambda\left(\varphi^{\epsilon} \cdot\right)$ & $1 \wedge(9 \cdot)$ & 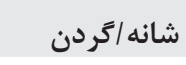 \\
\hline $\mathrm{P}=\cdot 1 \cdot 91$ & $q(\Gamma \cdot)$ & $I(\Delta)$ & $\operatorname{lf}(V \cdot)$ & $19(90)$ & يشت \\
\hline$P=1 / \cdots$ & $1(\Delta)$ & $\cdot(\cdot)$ & $19(90)$ & $r \cdot(1 \cdots)$ & اندام تحتانى \\
\hline
\end{tabular}




\begin{tabular}{|c|c|c|c|c|}
\hline \multicolumn{5}{|c|}{ جدول ه: ضريب همبستگى اسييرمن (مقدار احتمال)، بين نمره تجمعى ManTRA و شدت ناراحتى در اندامهاى مختلف بدن } \\
\hline \multirow[t]{2}{*}{ اندام تحتانى } & يشت & شانه/كردن & مج /دست/بازو & نمره تجمعى شدت درد \\
\hline & & & & سرو كردن \\
\hline$(\cdot / r q 1)-\cdot / 1 \uparrow \wedge$ & $(\cdot / \Lambda \cdot \vee)-\cdot / \cdot r \cdot$ & $(\cdot / V F \cdot)-\cdot / \cdot \Delta F$ & $(\cdot \mid \Lambda \cdot r) \cdot|\cdot| r \mid$ & ق ق قبل از مداخله \\
\hline \multirow[t]{2}{*}{$(\cdot / \mu \wedge \omega)-\cdot|| f \mid$} & $(\cdot / 9 \Lambda \uparrow)-\cdot / \cdot \cdot r$ & $(\cdot 10 \cdot 9) \cdot 11 \cdot v$ & $(\cdot / r \Delta \cdot) \cdot / l \Delta r$ & 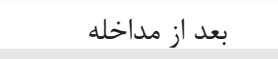 \\
\hline & & & & 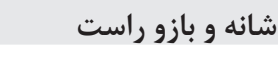 \\
\hline$(\cdot / 49 \Delta)-\cdot / 1111$ & $(\cdot / \Delta \cdot \Delta)-\cdot 11 \cdot 9$ & $(\cdot \mid 9 \cdot \cdot)-\cdot \cdot \cdot \wedge \Delta$ & $(\cdot 1991)-\cdot 1 \cdot V T$ & قبل از مداخله \\
\hline \multirow[t]{2}{*}{$(\cdot / r \cdot \Lambda) \cdot / 190$} & $(\cdot / r V r) \cdot / I V \Lambda$ & $(\cdot / \cdot 9 q) \cdot / 4 \& V$ & 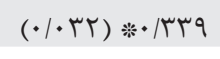 & بعد از مداخله \\
\hline & & & & شانه و بازو جي \\
\hline$(\cdot / r F \Delta)-\cdot / 1 \Delta r$ & $(\cdot / \mid V \cdot)-\cdot / 4 \mid \Lambda$ & $(\cdot / r F \Delta)-\cdot / l \Delta r$ & $(\cdot / 4 r \cdot)-\cdot / 1 r \Lambda$ & قبل از مداخله \\
\hline \multirow[t]{2}{*}{$(\cdot / r \cdot \Delta) \cdot / 199$} & $(\cdot / r \mid F) \cdot / r \cdot 1$ & $(\cdot / \cdot r \cdot) * \cdot r T V$ & $(\cdot / \cdot \wedge) * \cdot / 4 \mid \Delta$ & بعد از مداخله \\
\hline & & & & كمر \\
\hline$(\cdot / V \mid Y)-\cdot / \cdot 4$. & $(\cdot 1991) \cdot 1 \cdot V r$ & $(\cdot / 09 \Lambda) \cdot / \cdot 19$ & $(\cdot / F F T) \cdot / I T \Delta$ & قبل از مداخله \\
\hline \multirow[t]{2}{*}{$(\cdot / 119) \cdot / r \Delta 1$} & $(\cdot / \cdot F \wedge) * / r \mid Q$ & $(\cdot / \cdot r) * * / q q r$ & $(\cdot / \cdot r) * / r \wedge \Delta$ & بعد از مداخله \\
\hline & & & & آرنج و ساعد راست \\
\hline$(\cdot / \Delta \Delta r)-\cdot / \cdot 9 V$ & $(\cdot / 0 V I)-\cdot 1 \cdot 9 Y$ & $(\cdot / V Y \varepsilon)-\cdot / \cdot \Delta V$ & $(\cdot / \wedge V T)-\cdot / \cdot r q$ & قبل از مداخله \\
\hline \multirow[t]{2}{*}{$(\cdot / V q r) \cdot / \cdot q r$} & $(\cdot \mid q M \Lambda) \cdot / \cdot V \mathrm{~V}$ & $(\cdot / r r q) \cdot / / \Delta \Lambda$ & $(\cdot / r r \Delta) \cdot / 19 r$ & بعد از مداخله \\
\hline & & & & آرنج و ساعد جٍ \\
\hline$(\cdot / \cdot \vee q)-\cdot / r \wedge 1$ & $(\cdot / \cdot 9 \cdot)-\cdot / T V T$ & $(\cdot / R T)-\cdot / R T G$ & $(\cdot / r \wedge 9)-\cdot / 1 \vee r$ & قبل از مداخله \\
\hline \multirow[t]{2}{*}{$(\cdot / F \Delta I)-\cdot / 1 T H$} & $(\cdot \mid A \vee I)-\cdot \cdot \cdot r q$ & $(\cdot 1999) \cdot 1.94$ & $(\cdot|\Delta| r) \cdot / 1 \cdot V$ & بعد از مداخله \\
\hline & & & & دست و مجج راست \\
\hline$(\cdot / V q V)-\cdot / \cdot P r$ & $(\cdot / r r q)-\cdot / 190$ & $(\cdot / 4 \wedge \varepsilon)-\cdot / 11 \%$ & $(\cdot \mid A T I)-\cdot|| T \mid$ & قبل از مداخله \\
\hline \multirow[t]{2}{*}{$(\cdot / \cdot \Delta q) \cdot / r \cdot r$} & $(\cdot / r \cdot 9) \cdot / r \cdot r$ & $(\cdot / \cdot 1 \cdot) * \cdot / 4 \cdot r$ & $(\cdot / \cdot r) * / 4 \varepsilon$. & بعد از مداخله \\
\hline & & & & دست و مج جي \\
\hline$(\cdot / \cdot V \Delta)-\cdot / r \wedge \Delta$ & $(\cdot / \cdot F T) *-\cdot / T T H$ & $(\cdot / \cdot V 1)-\cdot / r \wedge 9$ & (ITI)-- & قبل از مداخله \\
\hline \multirow[t]{2}{*}{$(\cdot / r \cdot 1) \cdot / 19 \Lambda$} & $(\cdot|r| Y \mid) \cdot / 19$ & $(\cdot / \cdot r V) * \cdot r r r$ & $(\cdot / \cdot V) * / 4 T H$ & بعد از مداخله \\
\hline & & & & باسن \\
\hline$(\cdot / r \cdot 1)-\cdot / 1 r V$ & $(\cdot / \Delta \cdot 1)-\cdot / 1 \cdot 1$ & $(\cdot / \Lambda \wedge \varphi)-\cdot / \cdot r r$ & $(\cdot / V \mid F) \cdot / \cdot q$. & قبل از مداخله \\
\hline \multirow[t]{2}{*}{$(\cdot \mid \wedge \Delta \varphi)-\cdot / \cdot r \cdot$} & $(\cdot / V \wedge \Lambda)-\cdot / \cdot F q$ & $(\cdot / A V T) \cdot / \cdot T q$ & $(\cdot / V F V) \cdot / \cdot \Delta r$ & بعد از مداخله \\
\hline & & & & ران و زانو راست \\
\hline$(\cdot / \cdot V \Delta)-\cdot / r \wedge F$ & $(\cdot / \cdot \vee \Delta)-\cdot / r \wedge \Delta$ & $(\cdot|\cdot| r \wedge) *-\cdot|r| r$ & $(\cdot|\cdot r|) *-\cdot r q \mid$ & قبل از مداخله \\
\hline \multirow[t]{2}{*}{$(\cdot \mid \Lambda T F)-\cdot / \cdot r F$} & $(\cdot / F F V)-\cdot / I T F$ & $(\cdot / r q \cdot) \cdot / 1 \wedge r$ & $(\cdot / \Delta r \Delta)-\cdot / 1 \cdot r$ & بعد از مداخله \\
\hline & & & & ران و زانو جي \\
\hline$(\cdot / \cdot 19) *-\cdot / r V \Lambda$ & $(\cdot / \cdot \wedge) *-\cdot|4| \varphi$ & $(\cdot / \cdot r l) * \cdot / r g r$ & וTr|•-* & قبل از مداخله \\
\hline \multirow[t]{2}{*}{$(\cdot / T V V)-\cdot / I V g$} & $(\cdot \mid 191)-\cdot 14 r 9$ & $(\cdot / r \Delta T)-\cdot / / \wedge \Delta$ & $\left(\cdot|\Delta|^{F} \cdot\right)-\cdot / 1 \cdot \cdot$ & بعد از مداخله \\
\hline & & & & يا و ساق راست \\
\hline$(\cdot / 111)-\cdot / r \Delta s$ & $(\cdot / \cdot \wedge \varepsilon)-\cdot / r \vee v$ & $(\cdot / I T I)-\cdot / T F T$ & $(\cdot / \mid \Delta V)-\cdot / r Y \Lambda$ & قبل از مداخله \\
\hline \multirow[t]{2}{*}{$(\cdot|\Delta| 1)-\cdot \cdot 11 \cdot \Delta$} & $(\cdot / r q \varphi)-\cdot / / \mu \wedge$ & $(\cdot / F V F)-\cdot / 1 I V$ & $(\cdot / q \Gamma \Delta) \cdot / \cdot V V$ & بعد از مداخله \\
\hline & & & & يا و ساق جي \\
\hline$(\cdot / \cdot r q) * \cdot / r T \Lambda$ & $(\cdot / \cdot 9 \Delta)-\cdot / 199 \Delta$ & $(\cdot / \cdot r q) *-\cdot / r \top \Lambda$ & $(\cdot / \cdot \Delta \varphi)-\cdot / r \cdot \Delta$ & قبل از مداخله \\
\hline$(\cdot \mid \Lambda \Lambda T)-\cdot / \cdot r Y$ & $(\cdot \mid \Lambda \& V)-\cdot / \cdot Y V$ & $(\cdot|\Delta| \Lambda)-\cdot / 1 \cdot \Delta$ & $(\cdot \mid \wedge r \cdot)-\cdot \cdot \cdot r \Delta$ & بعد از مداخله \\
\hline
\end{tabular}

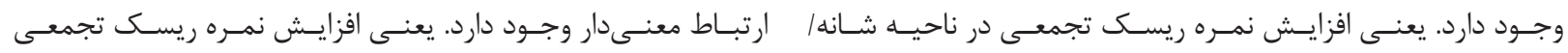

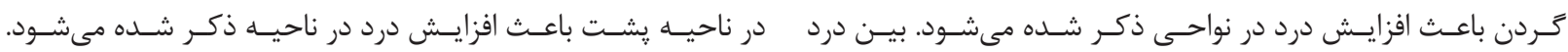

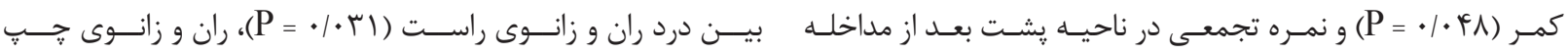




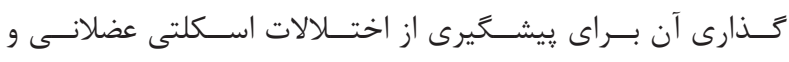

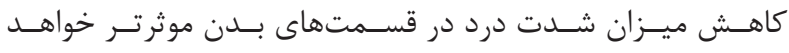

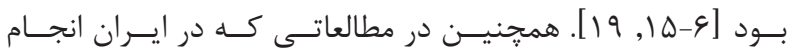

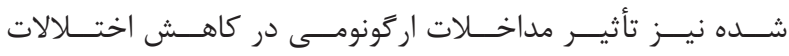

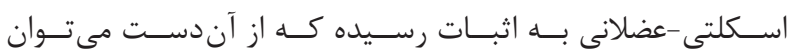

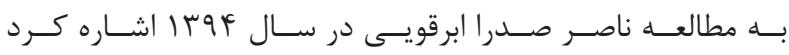

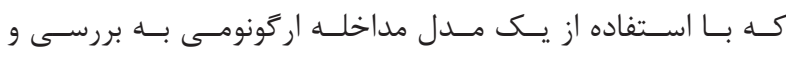

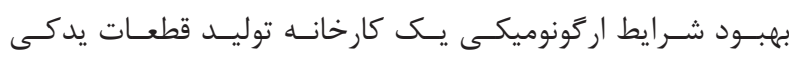

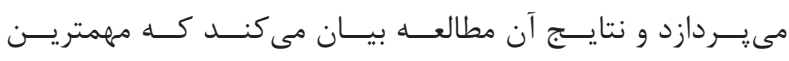

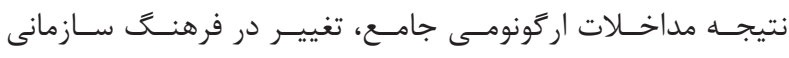

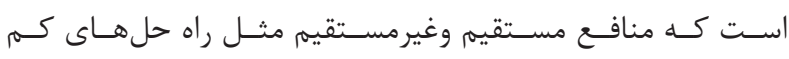

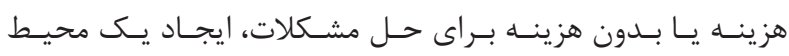

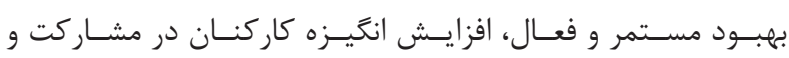

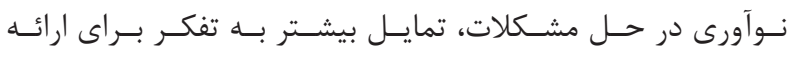

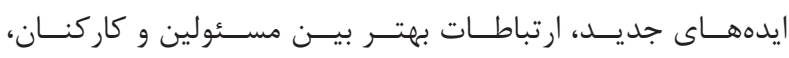

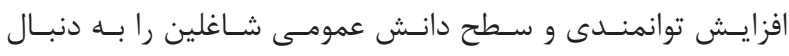

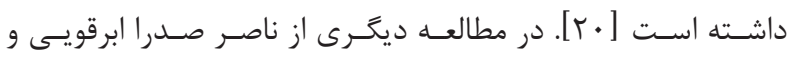

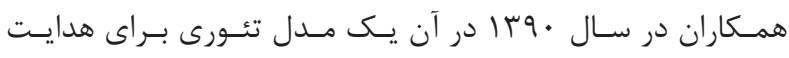

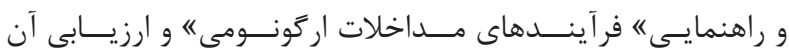

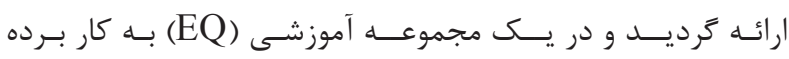

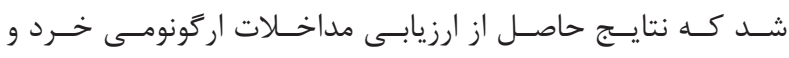

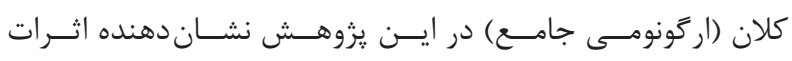

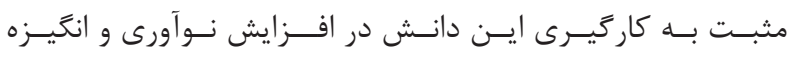

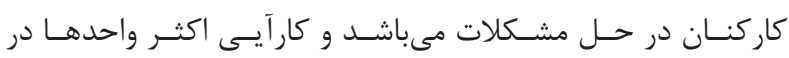

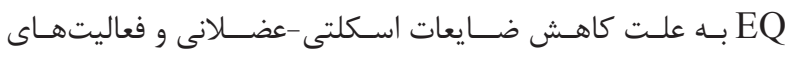

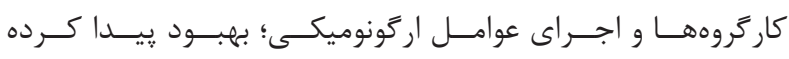

اسـت [rاركا

نتيجه كيرى

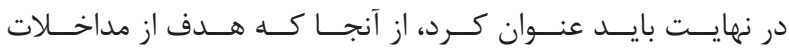

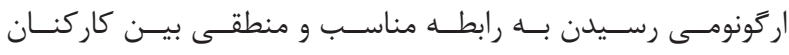

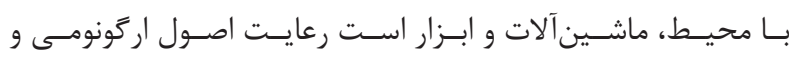

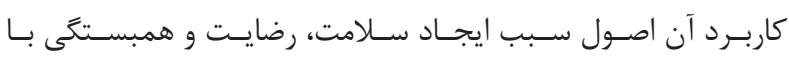

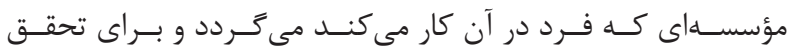

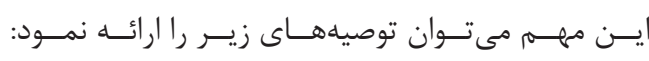

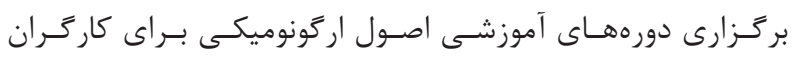

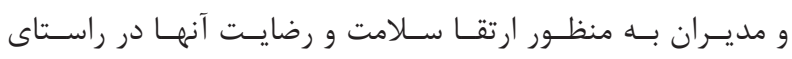

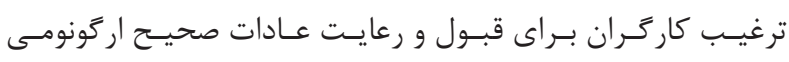

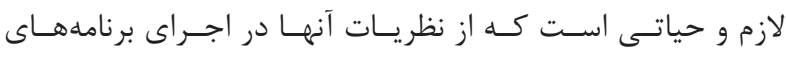

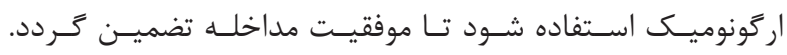

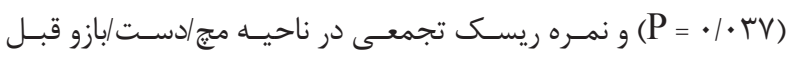

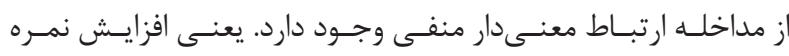

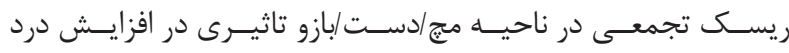

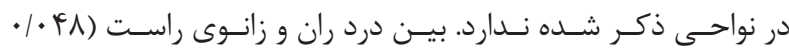

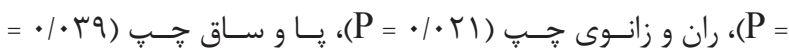

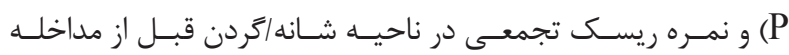

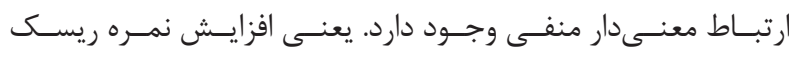

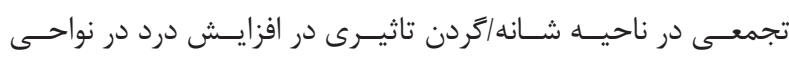

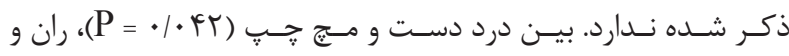

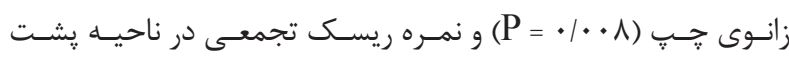

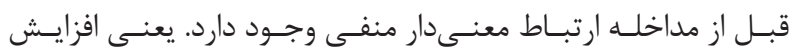

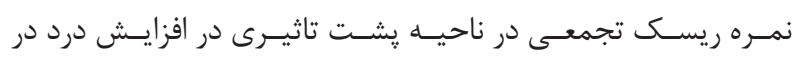

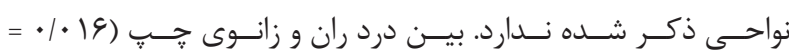

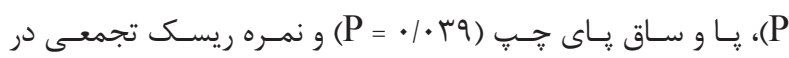

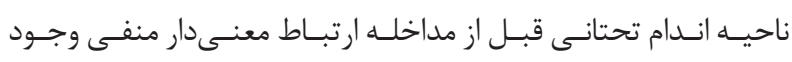

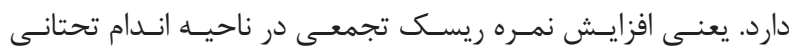

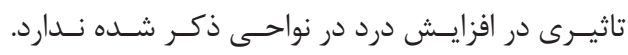

شـاخص ManTRA بــه عنــوان ابـززارى مفيـد، سـريع، آســان

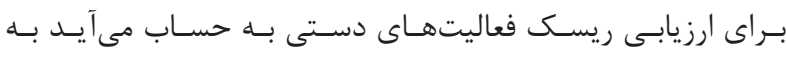

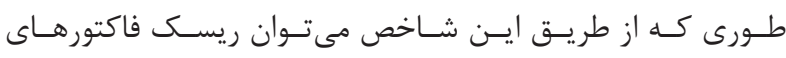

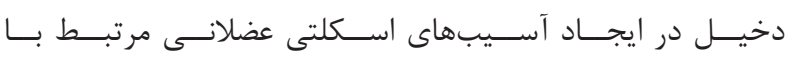

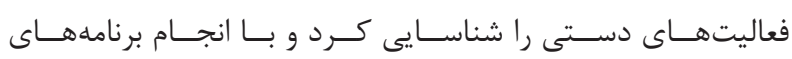

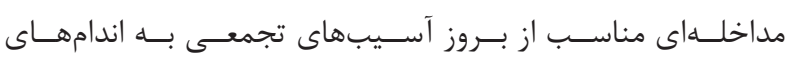

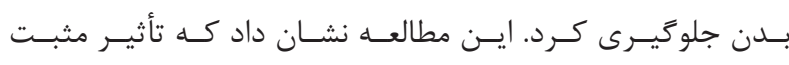

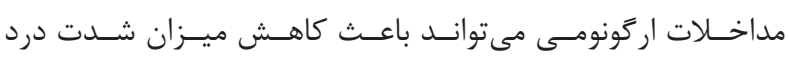

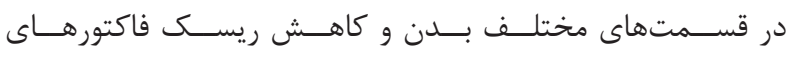

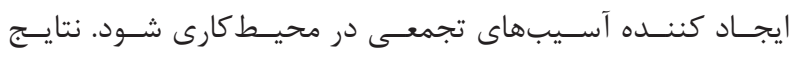

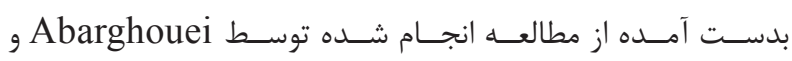
Hosseini Nasab

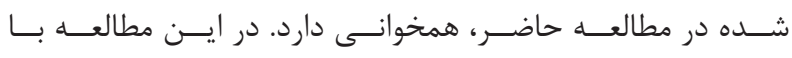

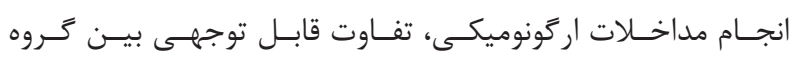

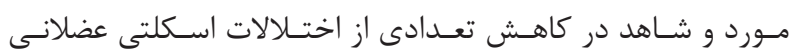

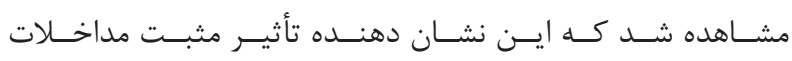

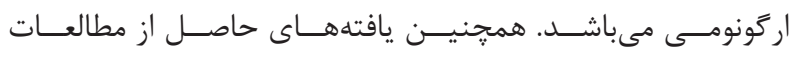

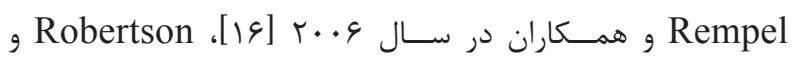

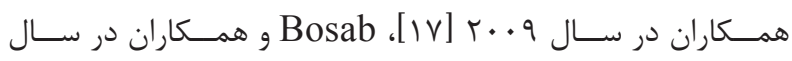

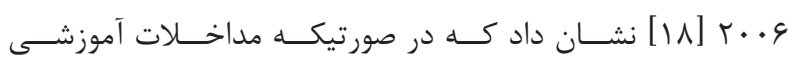

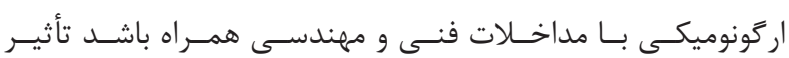




\section{REFERENCES}

1. Babakus E, Cravens DW, Johnston M, Moncrief WC. The Role of Emotional Exhaustion in Sales Force Attitude and Behavior Relationships. J Acad Mark Sci. 1999;27(1):58-70. DOI: $10.1177 / 0092070399271005$

2. Barling J, Loughlin C, Kelloway EK. Development and test of a model linking safety-specific transformational leadership and occupational safety. J Appl Psychol. 2002;87(3):488-96. PMID: 12090606

3. Nasl Saraji J, Malakuti J. [Documentation \& Auditing of Ergonomics Programs]. Fanavaran. 2013;1:13.

4. Ohlsson K, Attewell R, Skerfving S. Self-reported symptoms in the neck and upper limbs of female assembly workers. Impact of length of employment, work pace, and selection. Scand J Work Environ Health. 1989; 15(1):75-80. PMID: 2922592

5. Mattila M, Vilkki M. OWAS Methods. In: Karwowski W, Marras W, editors. The Occupational Ergonomics Handbook. Boca Raton, USA: CRC Press; 1999. p. 447-59.

6. Kilbom Å, Messing K. Work-related musculoskeletal disorders. Women Health Work. 2005:203-27.

7. Statistics BoL. Lost-work time Injuries and Illnesses: Characteristics and Resulting Time Away From Work. U.S: Department of Labor: Bureau of Labor Statistics, 2004.

8. Plawinski M, Vi P, Carlan N, Kramer D, Bigelow P, Wells R, et al. Using a manual tasks risk assessment tool (mantra) to audit the potential benefits of innovative interventions in the construction industry. 2009.

9. Straker L, Burgess-Limerick R, Pollock C, Egeskov R. A randomized and controlled trial of a participative ergonomics intervention to reduce injuries associated with manual tasks: physical risk and legislative compliance. Ergonomics. 2004;47(2):166-88. DOI: 10.1080/00140130310001617949 PMID: 14660211

10. Lai HS. Ergonomic Intervention to Reduce the Risk of Musculoskeletal Disorders (MSDs) for Manual Materials Handling Tasks. Malaysia Universiti Teknikal Malaysia Melaka; 2008.

11. Morken T, Moen B, Riise T, Hauge SHV, Holien S, Langedrag A, et al. Effects of a training program to improve musculoskeletal health among industrial workers - effects of supervisors role in the intervention. Int J Ind Ergonom. 2002;30(2):115-27. DOI: 10.1016/s01698141(02)00090-2
12. Hesam G, Motamedzade M, Moradpour Z. Ergonomics intervention in poultry slaughter industry and evaluate the effectiveness by key indicators method (KIM). J Ergonom. 2014;2(2):9-19.

13. Karwowski W, Marras W. Principles and application in engineering series occupational ergonomics engineering and administrative controls. New York: Taylor \& Francis; 2005.

14. Chobineh A. [Posture Assessment Practices In Occupational Ergonomics]. Tehran: Raven Press; 2005.

15. Takala EP, Pehkonen I, Forsman M, Hansson GA, Mathiassen SE, Neumann WP, et al. Systematic evaluation of observational methods assessing biomechanical exposures at work. Scand J Work Environ Health. 2010;36(1):3-24. PMID: 19953213

16. Rempel DM, Krause N, Goldberg R, Benner D, Hudes M, Goldner GU. A randomised controlled trial evaluating the effects of two workstation interventions on upper body pain and incident musculoskeletal disorders among computer operators. Occup Environ Med. 2006;63(5):300-6. DOI: 10.1136/oem.2005.022285 PMID: $\underline{16621849}$

17. Robertson M, Amick BC, 3rd, DeRango K, Rooney T, Bazzani $\mathrm{L}$, Harrist R, et al. The effects of an office ergonomics training and chair intervention on worker knowledge, behavior and musculoskeletal risk. Appl Ergon. 2009;40(1):124-35. DOI: 10.1016/i.apergo.2007.12.009 PMID: 18336791

18. Bos EH, Krol B, Van Der Star A, Groothoff JW. The effects of occupational interventions on reduction of musculoskeletal symptoms in the nursing profession. Ergonomics. 2006;49(7):706-23. DOI: $\underline{10.1080 / 00140130600578005}$ PMID: 16720530

19. Abarghouei NS, Nasab HH. An ergonomic evaluation and intervention model: macro ergonomic approach. Int J Sci Eng Res. $2012 ; 3(2): 1-7$.

20. Sadra Abarghouei N. [Comprehensive ergonomic interventions for improving ergonomic conditions in an automobile spare part manufacturing plant: A case study]. J Ergonom. 2015;3(2):1-13.

21. Sadra Abarqhouei N, Hosseini Nasab H, Fakhrzad M. [Macro Ergonomics Interventions and their Impact on Productivity and Reduction of Musculoskeletal disorders: Including a Case Study]. Iran Occup Health. 2012;9(2):27-39. 


\title{
The Effects of Ergonomic Interventions in Manual Activities to Reduce Musculoskeletal Disorders in Manual Activities by ManTRA
}

\author{
Sara Dehnavi ${ }^{1}$, Abdollah Vahedi ${ }^{2,}$, Majid Motamedzade ${ }^{3}$, Abas \\ Moghimbeigi $^{3}$
}

${ }^{1}$ Applied Science Education Center, Safa Industrial Group, Saveh, Iran

${ }^{2}$ Faculty of Engineering, Daneshestan Institute of Higher Education, Saveh, Iran

${ }^{3}$ Professor, Department of Ergonomics, School of Health and Research Center for

Health Sciences, Hamadan University of Medical Sciences, Hamadan, Iran

${ }^{4}$ Associate Professor, Department of Biostatistics \& Epidemiology, School of Public

Health, Hamadan University of Medical Sciences, Hamadan, Iran

* Corresponding author: Abdollah Vahedi, Faculty of Engineering, Daneshestan Insti-

DOI: $10.21859 /$ joe- 04048 tute of Higher Education, Saveh, Iran.E-mail: a.vahedi62@yahoo.com

Received: 12/07/2016

Accepted: $15 / 01 / 2017$

\section{Keywords:}

Musculoskeletal Disorders

ManTRA

Ergonomic Intervention

Manual Task

Body Map

\section{How to Cite this Article:}

Dehnavi S, Motamedzade M, Moghimbeigi A, Vahedi A. The Effects of Ergonomic Interventions in Manual Activities to Reduce Musculoskeletal Disorders in Manual Activities by ManTRA. $J$ Ergo. 2017;4(4):57-67. DOI: $10.21859 /$ joe -04048

(C) 2017 Hamedan University of Medical Sciences.

\section{Abstract}

Introduction: Work-related Musculoskeletal Disorders (WMSDs) occur when the pressure that applied to musculoskeletal tissue is more than tissue tolerant capabilities. This study aimed at assessing the risk factors that cause musculoskeletal disorders associated with manual tasks, using ManTRA, and conducting an ergonomic intervention program (educational engineering) among workers in the printing industry.

Methods: In this interventional case-control study, 40 workers, who performed manual tasks, (20 in the case group and 20 in the control group) were chosen and investigated. Nordic Body Map was used to determine the intensity and position of pain in different parts of the body. The ManTRA method was performed to evaluate cumulative scores in four regions of the body (wrist/hand/arm, shoulder/neck, back, and lower limb) for each worker before and after the intervention. Data analysis was done using the SPSS software. Results: Data analysis showed that mean intensity of pain in the control group before and after the intervention in various regions of the body, such as head/neck $(P=0.012)$, right shoulder/arm $(P=0.012)$, left shoulder/arm $(P=0.043)$, back $(P=0.003)$, right hand/ wrist $(\mathrm{P}=0.028)$, left hand/wrist $(\mathrm{P}=0.027)$, right knee/hip $(\mathrm{P}=0.005)$, left knee/hip $(\mathrm{P}$ $=0.005)$, right leg $(\mathrm{P}=0.012)$ and left leg $(\mathrm{P}=0.028)$ were significant. Cumulative score was also significant in body regions such as arm/wrist/hand $(\mathrm{P}=0.001)$ and shoulder/ neck $(\mathrm{P}=0.001)$ before and after the interventional program.

Conclusions: On the basis of the findings of this study, it could be noted that the ergonomic interventional program was effective for reducing the intensity of pain in various regions of the body and risk factors causing cumulative damage at the workplace. 